\title{
EXPONENTIAL TORSION GROWTH FOR RANDOM 3-MANIFOLDS
}

\author{
HYUNGRYUL BAIK, DAVID BAUER, ILYA GEKHTMAN, URSULA \\ HAMENSTÄDT, SEBASTIAN HENSEL, THORBEN KASTENHOLZ, BRAM PETRI, \\ DANIEL VALENZUELA
}

\begin{abstract}
We show that a random 3-manifold with positive first Betti number admits a tower of cyclic covers with exponential torsion growth.
\end{abstract}

\section{INTRODUCTION}

Given a manifold $M$ and a tower of coverings of $M$, i.e. a sequence

$$
\cdots \rightarrow M_{n} \rightarrow M_{n-1} \rightarrow \cdots \rightarrow M_{1} \rightarrow M
$$

of finite covers, one can ask about the growth of topological invariants for the manifolds in the sequence. In the case that $M$ is a hyperbolic 3 -manifold of finite volume, the study of such questions led to interesting conjectures which relate the growth of invariants of the sequence to invariants of hyperbolic 3-space.

More concretely, in the case of a tower of congruence covers of a closed arithmetic hyperbolic 3-manifold, conjecturally the growth rate of the torsion $H_{1}\left(M_{i}, \mathbb{Z}\right)_{\text {tor }}$ in the first homology group coincides with the $\ell^{2}$-torsion of $\mathbb{H}^{3}$, which equals $\frac{1}{6 \pi}$ (Conjecture 1.4 in BV13 proposes a slightly weaker statement).

Much earlier, torsion homology growth was studied for towers of abelian covers of knot complements. First results on the relation of this growth rate to the (logarithmic) Mahler measure of the Alexander polynomial of the knot or link can be found in Ri90 and GS91. Equality of this growth rate and the Mahler measure of the Alexander polynomial are due to Silver and Williams SW02a, and extensions of these results and an interpretation in the context of $\ell^{2}$-invariants can be found in SW02b] as well as in the more recent papers [BV13, Ra12, Le14.

As it became apparent in recent years, the existence of towers of covers with exponential torsion homology growth should be abundant for 3manifolds. The recent work [BGS16] explains that however, such towers do not exist for manifolds of higher dimension. The goal of this paper is

Date: March 10, 2017.

H. Baik, I. Gekhtman, U. Hamenstädt supported by ERC Advanced Grant "Moduli", B. Petri supported by the Max Planck Institut Bonn. AMS subject classification: 57M10 (57Q10). 
to study the existence of towers of cyclic covers with exponential torsion growth for random 3-manifolds in a sense that we make precise next.

Any closed 3-manifold $M$ admits a Heegaard decomposition. This means that $M$ can be obtained by gluing two handlebodies of some genus $g \geq 0$ with a diffeomorphism of their boundaries. The smallest genus of a handlebody which gives rise to $M$ in this way is called the Heegaard genus of $M$.

For a fixed base identification, the manifold $M$ only depends on the element in the mapping class group $\operatorname{Mod}\left(S_{g}\right)$ of the boundary surface $S_{g}$ of the handlebody defined by the gluing diffeomorphism. We denote by $N_{\varphi}$ the closed 3-manifold defined by the gluing $\operatorname{map} \varphi \in \operatorname{Mod}\left(S_{g}\right)$. Thus topological properties of closed 3 -manifolds $N_{\varphi}$ are directly related to properties of the mapping class $\varphi$.

This viewpoint was used by Dunfield and Thurston [DT06] to define the notion of a random 3-manifold using a random walk on the mapping class group. Embarking from [DT06, the purpose of this work is to study cyclic cover 11 of random hyperbolic 3-manifolds with positive first Betti number.

Let $\mathcal{I}_{\mathrm{g}}$ be the Torelli subgroup of $\operatorname{Mod}\left(S_{g}\right)$, i.e. the subgroup formed by all those mapping classes which act trivially on $H_{1}\left(S_{g} ; \mathbb{Z}\right)$. For $g \geq 3$ this is a finitely generated group. We use the following model for random 3 -manifolds with large Betti number, which is inspired by (but slightly different from) the Dunfield-Thurston model. Take any probability measure $\mu$ on $\mathcal{I}_{g}$ whose support equals a finite set which generates $\mathcal{I}_{g}$ as a semigroup. Such a $\mu$ defines a random walk on $\mathcal{I}_{g}$. We say that a property $\mathcal{P}$ holds for a random 3-manifold of Heegaard genus $g$ and maximal homology rank if the following holds: the proportion of 3-manifolds with $\mathcal{P}$ which are defined by a gluing with an element of the $n$-th step of the walk tends to one as $n \rightarrow \infty$, independently of $\mu$. To motivate this model, note that any 3 -manifold $M$ with Heegaard genus $g$ and first Betti number $b_{1}(M)=g$ is obtained as $N_{\varphi}$ for some $\varphi \in \mathcal{I}_{\mathrm{g}}$ (compare Section 2) and by [DT06] (see also [LMW14]), the Heegaard genus of a 3-manifold obtained from a random gluing in this sense is $g$. Furthermore, by a theorem by Maher [Ma10, a random manifold with Heegaard genus $g$ and maximal homology rank is indeed hyperbolic.

Theorem 1. A random 3-manifold of Heegaard genus $g \geq 3$ with maximal homology rank has a tower of cyclic covers with exponential torsion homology growth.

A precise version of this result is Theorem 6.1] in Section 6. We do not discuss the rate of convergence although we believe that it can be derived from careful analysis of Benoist and Quint's work on random walks on reductive groups BQ14, BQ16 (see also Section 7.6 of Ko08 and LMW14).

A result analogous to Theorem 1 remains true for random 3-manifolds with positive first Betti number (as opposed to maximal) by considering random walks not on the Torelli group $\mathcal{I}_{\mathrm{g}}$, but on a homology stabiliser which

\footnotetext{
${ }^{1}$ We always assume that cyclic covers are regular.
} 
is defined to be the subgroup of $\operatorname{Mod}(S)$ of all mapping classes preserving some fixed homology class.

Theorem 1 mainly is a result about the Torelli subgroup of the mapping class group. It does not rely on any information on the geometry and topology of hyperbolic 3-manifolds. Very recently, such topological/geometric tools were used by Liu [Li16] to construct for an arbitrary closed hyperbolic 3-manifold $M$ a tower of covers of $M$ with exponential torsion homology growth. These covers are however in general not regular. The existence of a finite cover $M^{\prime}$ of $M$ which admits a tower of cyclic covers with exponential torsion homology growth follows from Liu and Sun's beautiful virtual domination theorem [S15a, S15b, LiS16].

The methods used in the proof of Theorem 1 can also be used to show that the first Betti number of a random hyperbolic three-manifold $N_{\varphi}$ does not increase by passing to a finite Abelian cover of fixed degree.

Theorem 2. Fix a natural number $d>0$. A random 3-manifold of Heegaard genus $g \geq 3$ with maximal homology rank has no Abelian cover of degree $\leq d$ with Betti number $>g$.

A precise version of this result is Theorem 6.11 in Section 6. Theorem 9.1 in [DT06] shows that for a fixed number $k>0$, a random hyperbolic 3manifold of Heegaard genus two does not admit an Abelian cover of degree at most $k$ with positive first Betti number, where random refers to a random walk on the entire mapping class group. Our methods can be adapted to extend the result of DT06 to arbitrary genus, as outlined in the last section. We chose instead to focus on the version stated in Theorem 2 as its formulation is closer to the formulation of Theorem 1 .

As a final application of our methods, we also show that for a random walk on the full mapping class group, the order of $H_{1}\left(N_{\varphi}, \mathbb{Z}\right)_{\text {tors }}$ grows exponentially in the number of steps of the random walk (Theorem 6.14), answering a question of Kowalski [Ko08.

The proof of Theorem 1 relies on the relation between the growth rate of torsion in the homology for a tower of cyclic covers of the three-manifold $N_{\varphi}$ and the Mahler measure of the Alexander polynomial $\Delta$ of the corresponding infinite cyclic covering.

Given $\varphi \in \mathcal{I}_{\mathrm{g}}$, there is an infinite cyclic covering of $N_{\varphi}$ induced by an infinite cyclic covering $\widetilde{S}$ of the surface $S$. In Section 3 we give an explicit description of the homology of $\widetilde{S}$ as a $\mathbb{Z}[\mathbb{Z}]$-module. We construct a matrix $M(\varphi)$ with entries in the group ring $\mathbb{Z}[\mathbb{Z}]$ which describes the action of a lift of $\varphi$ on $H_{1}(\widetilde{S})$. In Section 5 we translate the condition that $\Delta$ has (logarithmic) Mahler measure 0 into a condition that is detectable by the action of lifts of $\varphi$ on finite covers $S_{q}$ of the surface $S$.

\footnotetext{
${ }^{2}$ In Ri14, Rivin claims this conclusion for solvable covers of random 3-manifolds of arbitrary Heegaard genus. However, his argument seems incomplete, although we believe that it can be completed in the Abelian case.
} 
Using an idea of Looijenga [Lo97, we then study the action of the Torelli group on the homology of these covers. The core result is Proposition 4.3. It shows that lifts of elements in the Torelli group generate a dense subgroup of an algebraic group which is closely related to the automorphism group of the homology of some finite cyclic cover of $S$. Using results of Benoist and Quint BQ14 on random walks on algebraic groups, we then deduce that random elements in $\mathcal{I}_{\mathrm{g}}$ with probability one violate the conditions implied by $\Delta$ having Mahler measure zero.

The main novelty of our approach lies in a direct translation of properties of random walks on the Torelli group into properties of random walks on algebraic groups. We do not use any of the recent results on random walks on the mapping class group.

Acknowledgements: This work was carried out in fall 2015 while all authors were in residence in Bonn. All of us thank Gregor Masbaum for useful conversations. Sebastian Hensel is grateful to Benson Farb for helpful discussions. We are particularly grateful to an anonymous referee who pointed out an error in an earlier version of this paper and whose suggestions led moreover to a significant simplification of our argument.

\section{Covers of Surfaces And 3-Manifolds}

In this section we describe the setup we will use to determine and control the homology of covers of 3-manifolds given by Heegaard splittings. The terminology introduced in this section will be used throughout the article.

Let $S$ be a surface of genus $g \geq 2$, identified once and for all with the boundary $\partial V=S$ of a handlebody $V$. Such a handlebody is a compact manifold with boundary which is homeomorphic to the thickening of an bouquet of $g$ circles embedded in $\mathbb{R}^{3}$. A meridian of $V$ is an essential simple closed curve on $S$ which bounds a disk in $V$.

Let $\alpha_{1}, \ldots, \alpha_{g}$ be a set of (oriented) simple closed curves in $S$ which form a cut system for $V$. This means that the curves $\alpha_{i}$ are pairwise disjoint meridians for $V$ whose complement $S-\cup_{i} \alpha_{i}$ is connected. In particular, the $\alpha_{i}$ are pairwise non-homologous and all non-separating. Then

$$
L=\operatorname{ker}\left(H_{1}(S ; \mathbb{Z}) \rightarrow H_{1}(V ; \mathbb{Z})\right)=\operatorname{span}_{\mathbb{Z}}\left\{\left[\alpha_{1}\right], \ldots,\left[\alpha_{g}\right]\right\},
$$

and $L$ is a Lagrangian subspace of $H_{1}(S, \mathbb{R})$ with respect to the algebraic intersection pairing

$$
(\cdot, \cdot): H_{1}(S, \mathbb{R}) \times H_{1}(S, \mathbb{R}) \rightarrow \mathbb{R}
$$

on homology.

Let $\beta_{1}, \ldots, \beta_{g}$ be a set of simple closed curves on $S$ dual to the cut system $\left\{\alpha_{i}\right\}$. This means that the curves $\beta_{i}$ are pairwise disjoint (and transverse to the curves $\alpha_{j}$ for some smooth structure), and $\#\left(\alpha_{i} \cap \beta_{j}\right)=\delta_{i j}$. We assume that the $\alpha_{i}, \beta_{j}$ are oriented so that $\left(\alpha_{i}, \beta_{j}\right) \geq 0$ for all $i, j$. The $\alpha_{i}, \beta_{i}$ project to a symplectic basis $a_{1}, \ldots, a_{g}, b_{1}, \ldots, b_{g}$ of $H_{1}(S ; \mathbb{Z})$ (here $a_{i}=\left[\alpha_{i}\right]$ for the above notation). 
Given any mapping class $\varphi \in \operatorname{Mod}(S)$ of $S$, we denote by $N_{\varphi}$ the 3manifold given by the Heegaard splitting defined by $\varphi$ :

$$
N_{\varphi}=V \cup_{\varphi} V
$$

With our convention, the identity mapping class gives rise to the manifold $N_{\text {id }}=S^{2} \times S^{1} \sharp \ldots \sharp S^{2} \times S^{1}$ ( $g$ copies). We have (see the beginning of Section 8 of [DT06]).

\section{Lemma 2.1.}

$$
H_{1}\left(N_{\varphi} ; \mathbb{Z}\right)=H_{1}(S ; \mathbb{Z}) /\left\langle L, \varphi_{*} L\right\rangle
$$

where $\varphi_{*}$ denotes the induced map of $\varphi$ on homology.

The following lemma relates the first Betti number $b_{1}\left(N_{\varphi}\right)$ of $N_{\varphi}$ to information on the gluing map $\varphi$. For its statement, we need to introduce certain subgroups of $\operatorname{Mod}(S)$. The Torelli group $\mathcal{I}_{\mathrm{g}}$ is the group of all mapping classes acting trivially on $H_{1}(S ; \mathbb{Z})$. The handlebody group is the subgroup $\mathcal{H}_{g}$ of $\operatorname{Mod}(S)$ of those mapping classes which can be represented by diffeomorphisms of $S$ extending to $V$. Finally, given homology classes $a, b \in H_{1}(S ; \mathbb{Z})$ we denote the homology stabiliser group by

$$
H S(a, b)=\left\{f \in \operatorname{Mod}(S) \mid f_{*}(a)=a, f_{*}(b)=b\right\}
$$

Note that $\mathcal{I}_{\mathrm{g}}<H S(a, b)$ for any $a, b$.

Lemma 2.2. a) $b_{1}\left(N_{\varphi}\right) \leq g$ with equality if and only if $\varphi=\psi_{1} \psi_{2}$ where $\psi_{1} \in \mathcal{I}_{\mathrm{g}}, \psi_{2} \in \mathcal{H}_{g}$.

b) $b_{1}\left(N_{\varphi}\right) \geq 1$ if and only if there are $a \in L, b \in H_{1}(S ; \mathbb{Z}),(a, b)=1$ so that $\varphi=\psi_{1} \psi_{2}$ with $\psi_{1} \in H S(a, b), \psi_{2} \in \mathcal{H}_{g}$.

Proof. Lemma 2.1 shows that the first Betti number of $N_{\varphi}$ is at most $g$. The same simple observation which leads to Lemma 2.1 (see the discussion in Section 8 of [DT06]) also yields that the conditions in a), b) are sufficient for the Betti number bound. We proceed to show necessity.

a) By Lemma 2.1, if $b_{1}\left(N_{\varphi}\right)=g$ then $\varphi_{*} L=L$. In other words, the matrix describing $\varphi_{*}$ with respect to the symplectic basis introduced above has the form

$$
\left(\begin{array}{ll}
A & B \\
0 & C
\end{array}\right)
$$

Now any symplectic matrix of such a form is induced by an element of the handlebody group [Hi06]. The claim follows.

b) By Lemma 2.1, if $b_{1}\left(N_{\varphi}\right) \geq 1$ then there is some $0 \neq v \in L \cap \varphi_{*} L$. Since $\varphi_{*}$ is an automorphism of $H_{1}(S, \mathbb{Z})$ we may assume that $v$ is primitive (which is equivalent to stating that $v$ can be represented by a simple closed curve, see [FM12, Proposition 6.2]). Since $\mathcal{H}_{g}$ acts transitively on the set of primitive vectors in $L$, by multiplying $\varphi$ from the right by an element in $\mathcal{H}_{g}$ we may assume that $\varphi_{*}(v)=v$. Using the description of the image of the handlebody group in $\operatorname{Sp}(2 g, \mathbb{Z})$ given above Hi06, it follows that the stabiliser in $\mathcal{H}_{g}$ of an element $v \in L$ acts transitively on 
the set of primitive vectors $w \in H_{1}(S ; \mathbb{Z})$ with $(v, w)=1$. The claim follows.

Next we discuss how covers of $S$ give rise to covers of $N_{\varphi}$. The following easy lemma can also be found in DT06 $^{3}$.

Lemma 2.3. Let $\sigma: \pi_{1}(S) \rightarrow G$ be a surjection onto a group $G$. Then $\sigma$ factors through a map $\pi_{1}\left(N_{\varphi}\right) \rightarrow G$ if and only if

$$
K=\operatorname{ker}\left(\pi_{1}(S) \rightarrow \pi_{1}(V)\right) \subset \operatorname{ker}(\sigma) \text { and } \varphi_{*} K \subset \operatorname{ker}(\sigma) .
$$

Proof. This is an immediate consequence of the fact that

$$
\pi_{1}\left(N_{\varphi}\right)=\pi_{1}(S) /\left\langle\left\langle K, \varphi_{*} K\right\rangle\right\rangle
$$

where $\left\langle\left\langle K, \varphi_{*} K\right\rangle\right\rangle$ denotes the normal closure of the subgroup of $\pi_{1}(S)$ generated by $K, \varphi_{*} K$. The former statement can e.g. be derived from the theorem of Seifert-van Kampen.

In particular, we have the following.

Corollary 2.4. Let $\sigma: \pi_{1}(S) \rightarrow G$ be a surjection onto an Abelian group $G$ so that $K=\operatorname{ker}\left(\pi_{1}(S) \rightarrow \pi_{1}(V)\right) \subset \operatorname{ker}(\sigma)$. Denote by $S^{\prime}$ the cover of $S$ defined by $\sigma$. Let $\varphi \in \mathcal{I}_{\mathrm{g}}$ be arbitrary. Then:

i) $S^{\prime}=\partial V^{\prime}$ for a cover $V^{\prime}$ of $V$, and the action of the deck group $G$ on $S^{\prime}$ extends to the action of the deck group of $V^{\prime} \rightarrow V$.

ii) $\sigma$ factors through a map $\sigma_{\varphi}: \pi_{1}\left(N_{\varphi}\right) \rightarrow G$.

iii) The cover $\widetilde{N}_{\varphi} \rightarrow N_{\varphi}$ defined by $\sigma_{\varphi}$ is homeomorphic to $N_{\widetilde{\varphi}}$, where $\widetilde{\varphi}$ is any lift of $\varphi$ to $S^{\prime}$.

The same remains true if $\varphi \in H S(a, b)$, assuming that $\sigma: \pi_{1}(S) \rightarrow G$ is defined by algebraic intersection number (possibly mod $q>0$ ) with a.

Proof. To show the first assertion, suppose that $K \subset \operatorname{ker}(\sigma)$ and let $V^{\prime} \rightarrow V$ be the cover of the handlebody $V$ whose fundamental group is the image $\operatorname{ker}(\sigma) / K$ in $\pi_{1}(V)$ of the subgroup $\operatorname{ker}(\sigma)<\pi_{1}(S)$. Note first that

$$
G=\pi_{1}(S) / \operatorname{ker}(\sigma)=\left(\pi_{1}(S) / K\right) /(\operatorname{ker}(\sigma) / K)
$$

and hence $V^{\prime} \rightarrow V$ is a regular cover with deck group $G$. The induced cover $\partial V^{\prime} \rightarrow \partial V=S$ has fundamental group exactly $\operatorname{ker}(\sigma)$, and therefore it is equal to $S^{\prime} \rightarrow S$.

The second statement is immediate from Lemma 2.3 and the fact that $\sigma$ factors through a homomorphism $H_{1}(S, \mathbb{Z}) \rightarrow G$. In particular, any element of $\mathcal{I}_{\mathrm{g}}$ lifts to $V^{\prime}$, and this lift commutes with the action of $G$ which implies the third statement. Under the extra assumption given at the end, the same is true for $\varphi \in H S(a, b)$.

\footnotetext{
${ }^{3}$ In the terminology of [DT06]: a map $\sigma$ induces a cover of $N_{\varphi}$ if and only if $\sigma$ extends over $V$ and $\varphi \cdot \sigma$ extends over $V$.
} 
We call a cover $S^{\prime} \rightarrow S$ as in Corollary 2.4 a $K$-cover. For any $K$-cover, by part i) of that corollary, there is a subspace

$$
L^{\prime}=\operatorname{ker}\left(H_{1}\left(S^{\prime} ; \mathbb{Z}\right) \rightarrow H_{1}\left(V^{\prime} ; \mathbb{Z}\right)\right)
$$

and thus, by Lemma 2.1, we obtain

Proposition 2.5. With notation as above, we have

$$
H_{1}\left(\widetilde{N_{\varphi}} ; \mathbb{Z}\right)=H_{1}\left(N_{\widetilde{\varphi}} ; \mathbb{Z}\right)=H_{1}\left(S^{\prime} ; \mathbb{Z}\right) /\left\langle L^{\prime}, \widetilde{\varphi}_{*} L^{\prime}\right\rangle
$$

where $\widetilde{\varphi}$ is any lift of $\varphi$ to $S^{\prime}$.

We will also need the following version which is useful to compare Betti numbers. To this end, define

$$
E=\operatorname{ker}\left(H_{1}\left(S^{\prime} ; \mathbb{Z}\right) \rightarrow H_{1}(S ; \mathbb{Z})\right)
$$

and let $L_{E}^{\prime}=L^{\prime} \cap E$. By transfer, we have

$$
H_{1}\left(S^{\prime} ; \mathbb{Q}\right)=H_{1}(S ; \mathbb{Q}) \oplus(E \otimes \mathbb{Q}) .
$$

Furthermore, $E$ and this decomposition is preserved by $\widetilde{\varphi}_{*}$. This, together with Proposition 2.5 yields the following useful characterisation.

Proposition 2.6. With notations as above, we have

$$
H_{1}\left(\widetilde{N_{\varphi}} ; \mathbb{Q}\right)=H_{1}\left(N_{\varphi} ; \mathbb{Q}\right) \oplus\left(E \otimes \mathbb{Q} /\left\langle L_{E}^{\prime} \otimes \mathbb{Q}, \widetilde{\varphi}_{*} L_{E}^{\prime} \otimes \mathbb{Q}\right\rangle\right)
$$

In particular, $b_{1}\left(\widetilde{N_{\varphi}}\right)>b_{1}\left(N_{\varphi}\right)$ if and only if

$$
L_{E}^{\prime}+\widetilde{\varphi}_{*} L_{E}^{\prime} \subsetneq E
$$

is not a lattice.

In the sequel, two special kinds of $K$-covers will be particularly important. Namely, given any primitive vector $a \in L$, the kernel of the map which associates to an element $\alpha \in \pi_{1}(S)$ the algebraic intersection number with $a$ of the homology class defined by $\alpha$ (resp. its algebraic intersection number with $a \bmod q$ for $q \in \mathbb{N}$ ) defines an infinite cyclic cover $S_{\infty} \rightarrow S$ (resp. a cyclic cover $S_{q} \rightarrow S$ of order $q$ ). Informally, we call such a cover the cover defined by algebraic intersection number with $a$.

By Lemma 2.2, if $N_{\varphi}$ has Betti number at least 1, then a may be chosen such that these covers induce covers of the $3-$ manifold $N_{\varphi}$. In the sequel we always do so.

\section{CyClic Covers}

Recall from Section 2 the choice $a_{1}, \ldots, a_{g}, b_{1}, \ldots, b_{g}$ of a symplectic basis of $H_{1}(S, \mathbb{Z})$. The classes $a_{1}, \ldots, a_{g}$ generate the kernel $L$ of the map $H_{1}(S ; \mathbb{Z}) \rightarrow H_{1}(V ; \mathbb{Z})$ induced by the inclusion $S=\partial V \rightarrow V$. Let $S_{\infty} \rightarrow S$ be the infinite cyclic $K$-cover of $S$ defined by algebraic intersection number with $a_{g}$. There is a corresponding infinite cyclic cover $V_{\infty} \rightarrow V$. Since linear functionals on $H_{1}(S, \mathbb{Z})$ defined by algebraic intersection with nonseparating simple closed curves generate $H^{1}(S ; \mathbb{Z})$ and the mapping class 
group acts transitively on such curves, the results in this section in fact hold true for any cyclic $K$-cover $S_{\infty} \rightarrow S$. We will restrict to the case of intersection with $a_{g}$ for clarity.

3.1. Homology of the Infinite Cyclic Cover. The goal of this subsection is to give a fairly explicit (but non-canonical) description of the first homology of $S_{\infty}$ as a module over the group ring $\mathbb{Z}[\mathbb{Z}]$ of the deck group $\mathbb{Z}$ of $S_{\infty}$.

Denote by $Y \subset S$ the complementary subsurface of the simple closed curve $\alpha_{g}$ on $S$. Choose a preferred lift $\widetilde{Y}$ of $Y$ to $S_{\infty}$, i.e. $\tilde{Y} \subset S_{\infty}$ is a connected subsurface with boundary which is mapped by the covering map $S_{\infty} \rightarrow S$ homeomorphically onto $Y$.

For $i, j \leq g-1$ the curves $\alpha_{i}, \beta_{j}$ (defining the homology classes $a_{i}, b_{j}$ ) admit unique lifts

$$
\widetilde{\alpha}_{1}, \ldots, \widetilde{\alpha}_{g-1}, \widetilde{\beta}_{1}, \ldots, \widetilde{\beta}_{g-1}
$$

to $\widetilde{Y}$. Let $\widetilde{\alpha}_{g}$ be the lift of $\alpha_{g}$ contained in the closure of $\widetilde{Y}$ whose orientation agrees with the boundary orientation of $\widetilde{Y}$. Note that this makes sense since the orientation of $S$ induces an orientation of $\tilde{Y}$ and since $\alpha_{g}$ is an oriented curvet. Denote by $\widetilde{a}_{i}, \widetilde{b}_{j}(1 \leq i \leq g, 1 \leq j \leq g-1)$ the homology classes in $H_{1}\left(S_{\infty} ; \mathbb{Z}\right)$ of the curves $\widetilde{\alpha}_{i}, \widetilde{\beta}_{j}$. We have an isomorphism $H_{1}(Y ; \mathbb{Z}) \rightarrow$ $H_{1}(\widetilde{Y} ; \mathbb{Z})$ which sends $a_{i}, b_{j}$ to $\widetilde{a}_{i}, \widetilde{b}_{j}$.

As usual, we denote by $\mathbb{Z}[G]$ the integral group ring of a group $G$. Then $\mathbb{Z}[\mathbb{Z}]$ has an obvious identification with the ring $\mathbb{Z}\left[t, t^{-1}\right]$ of integral Laurent polynomials. If $H$ is a $\mathbb{Z}$-module, we write $H\left[t, t^{-1}\right]$ to mean $H \otimes_{\mathbb{Z}} \mathbb{Z}[\mathbb{Z}]$. We also choose $\tau$ a generator of the deck group of $S_{\infty}$.

Lemma 3.1. The map

$$
H_{1}(Y ; \mathbb{Z})\left[t, t^{-1}\right] \oplus \mathbb{Z} \cong \mathbb{Z}[\mathbb{Z}]^{2 g-2} \oplus \mathbb{Z} \rightarrow H_{1}\left(S_{\infty} ; \mathbb{Z}\right)
$$

induced by sending $a_{i}$ to $\widetilde{a}_{i}, b_{i}$ to $\widetilde{b}_{i}$ for $i<g$ and the generator of the trivial $\mathbb{Z}[\mathbb{Z}]$-module $\mathbb{Z}$ to $\widetilde{a}_{g}$ is an isomorphism of $\mathbb{Z}[\mathbb{Z}]$-modules.

Furthermore, the image of $L\left[t, t^{-1}\right] \oplus \mathbb{Z}$ under this map is exactly

$$
\operatorname{ker}\left(H_{1}\left(S_{\infty} ; \mathbb{Z}\right) \rightarrow H_{1}\left(V_{\infty} ; \mathbb{Z}\right)\right)
$$

Proof. Recall that $\widetilde{Y}$ is homeomorphic to $Y$, and that the subsurfaces $\tau^{n} \widetilde{Y}$ are pairwise disjoint $(n \in \mathbb{Z})$. The integral homology of $Y$ equals $\mathbb{Z}^{2 g-1}=$ $F \oplus \mathbb{Z} \tilde{a}_{g}$ where the free $\mathbb{Z}$-module $F$ of rank $2 g-2$ is spanned by the classes $\tilde{a}_{i}, \tilde{b}_{j}(1 \leq i, j \leq g-1)$.

The closures of $\tau^{n} \widetilde{Y}$ and $\tau^{n+1} \widetilde{Y}$ intersect in the lift $\tau^{n} \widetilde{\alpha_{g}}$ of $\alpha_{g}$. Enlarging slightly the set $\tilde{Y}$ to a neighborhood of its closure allows to apply the Mayer Vietoris sequence to $\cup_{i} \tau^{i} \widetilde{Y}$ to calculate the homology of $S_{\infty}$. We find that

$$
H_{1}\left(S_{\infty} ; \mathbb{Z}\right)=\Pi\left(\oplus_{i \in \mathbb{Z}} H_{1}\left(\tau^{i} \tilde{Y} ; \mathbb{Z}\right)\right)
$$

\footnotetext{
${ }^{4}$ This just serves to fix a specific lift, we could take any.
} 
where the map $\Pi$ is the identity on $\oplus_{i} \tau^{i} F$ and identifies $\tau^{i} \tilde{a}_{g}$ with $\tau^{i+1} \tilde{a}_{g}$.

Now observe that the deck group $\mathbb{Z}$ acts on both sides of this equation, and that the map $\Pi$ is equivariant for this action. Thus $H_{1}\left(S_{\infty} ; \mathbb{Z}\right)=\mathbb{Z}[\mathbb{Z}]^{2 g-2} \oplus$ $(\mathbb{Z}[\mathbb{Z}] / \operatorname{ker}(\varepsilon))$ where $\varepsilon: \mathbb{Z}[\mathbb{Z}] \rightarrow \mathbb{Z}$ is the $\mathbb{Z}$-linear map defined by $\varepsilon(g)=1$ for all $g \in \mathbb{Z}$. This shows the first assertion of the lemma.

The second assertion follows from the fact that the $\alpha_{i}$ normally generate the kernel of $\pi_{1}(S) \rightarrow \pi_{1}(V)$.

We define

$$
F_{\infty}=\operatorname{span}_{\mathbb{Z}[\mathbb{Z}]}\left(\widetilde{a}_{1}, \ldots, \widetilde{a}_{g-1}, \widetilde{b}_{1}, \ldots, \widetilde{b}_{g-1}\right)
$$

and note that it is a free $\mathbb{Z}[\mathbb{Z}]$-submodule of $H_{1}\left(S_{\infty} ; \mathbb{Z}\right)$. We will frequently use the decomposition

$$
\begin{gathered}
F_{\infty}=C_{\infty} \oplus D_{\infty} \\
D_{\infty}=\operatorname{span}_{\mathbb{Z}[\mathbb{Z}]}\left\{\widetilde{a}_{i}, i<g\right\}, \quad C_{\infty}=\operatorname{span}_{\mathbb{Z}[\mathbb{Z}]}\left\{\widetilde{b}_{i}, i<g\right\} .
\end{gathered}
$$

By the above discussion, both $C_{\infty}$ and $D_{\infty}$ are free $\mathbb{Z}[\mathbb{Z}]$-modules of rank $g-1$.

\subsection{Matrices.}

Lemma 3.2. Suppose $\varphi \in H S\left(a_{g}, b_{g}\right)$. Then $\varphi$ lifts to $S_{\infty}$, the lift commutes with the deck group action, and the induced map $\widetilde{\varphi}_{*}$ on homology preserves $F_{\infty}$

Proof. The fact that $\varphi$ lifts is immediate from the fact that $H S\left(a_{g}, b_{g}\right)$ preserves algebraic intersection number with $a_{g}$. The same fact also implies that any lift of $\varphi$ commutes with the deck group action.

We are left with showing the last claim. Let as before $\tau$ be a generator of the deck group of $S_{\infty}$. By Lemma 3.1, for any $i<g$ there are numbers $n_{k, j}^{a}, n_{k, j}^{b}, m \in \mathbb{Z}$ so that

$$
\widetilde{\varphi}_{*}\left(\widetilde{a}_{i}\right)=\sum_{j<g, k \in \mathbb{Z}} n_{k, j}^{a} \tau^{k}{\widetilde{a_{j}}}+m \widetilde{a}_{g}+\sum_{j<g, k \in \mathbb{Z}} n_{k, j}^{b} \tau^{k} \widetilde{b}_{j} .
$$

Thus we have

$$
\varphi_{*}\left(a_{i}\right)=\sum_{j<g, k \in \mathbb{Z}} n_{k, j}^{a} a_{j}+m a_{g}+\sum_{j<g, k \in \mathbb{Z}} n_{k, j}^{b} b_{j}
$$

Since $\varphi \in H S\left(a_{g}, b_{g}\right)$ we compute

$$
m=\left(\varphi_{*}\left(a_{i}\right), b_{g}\right)=\left(\varphi_{*}\left(a_{i}\right), \varphi_{*}\left(b_{g}\right)\right)=\left(a_{i}, b_{g}\right)=0 .
$$

This implies $\widetilde{\varphi}_{*}\left(\widetilde{a}_{i}\right)=\sum_{j<g, k \in \mathbb{Z}} n_{k, j}^{a} \tau^{k} \widetilde{a}_{j}+\sum_{j<g, k \in \mathbb{Z}} n_{k, j}^{b} s \tau^{k} \widetilde{b}_{j} \in F_{\infty}$. The case of $\widetilde{\varphi}_{*}\left(\widetilde{b}_{i}\right)$ is similar.

Definition 3.3. Let $\varphi \in H S\left(a_{g}, b_{g}\right)$. 
i) Denote by $M_{\infty}(\varphi)$ the $(2 g-2) \times(2 g-2)$-matrix with entries in $\mathbb{Z}[\mathbb{Z}]$ describing the action of a lift $\widetilde{\varphi}_{*}$ of $\varphi$ on $F_{\infty}$, with respect to the basis $\widetilde{a}_{1}, \ldots, \widetilde{a}_{g-1}, \widetilde{b}_{1}, \ldots, \widetilde{b}_{g-1}$. The matrix $M_{\infty}(\varphi)$ is well-defined up to a unit in $\mathbb{Z}[\mathbb{Z}]$ (namely, it depends on the choice of a lift of $\varphi$, any two of which differ by multiplication with some $\left.t^{k}\right)$.

ii) Denote by $B_{\infty}(\varphi)$ the "bottom-left block" of $M_{\infty}(\varphi)$; explicitly, $B_{\infty}(\varphi)$ is the $(g-1) \times(g-1)$-matrix defined by the requirement that for $j<g$

$$
\widetilde{\varphi}_{*}\left(\widetilde{a}_{j}\right)=d+\sum_{i=1}^{g-1}\left(B_{\infty}(\varphi)\right)_{i, j} \widetilde{b}_{i}
$$

where $d \in D_{\infty}$. Again, $B_{\infty}(\varphi)$ is only well-defined up to a unit in $\mathbb{Z}[\mathbb{Z}]$ (in the same sense as above).

Similarly, for $\varphi \in H S\left(a_{g}, b_{g}\right)$ the determinant

$$
\operatorname{det} B_{\infty}(\varphi) \in \mathbb{Z}[\mathbb{Z}]
$$

is defined up to a unit in $\mathbb{Z}[\mathbb{Z}]$. We will usually assume that the unit is chosen so that $\operatorname{det} B_{\infty}(\varphi)$ is a polynomial.

Remark 3.4. If $\varphi \in \mathcal{I}_{\mathrm{g}}$ is an element of the Torelli group, then we can write $M_{\infty}(\varphi)=\mathrm{Id}+M_{\infty}^{\prime}(\varphi)$ where $M_{\infty}^{\prime}(\varphi)$ is a matrix with entries in the augmentation ideal $\mathfrak{a}=\operatorname{ker}(\mathbb{Z}[\mathbb{Z}] \rightarrow \mathbb{Z})$. This can be proved with an argument very much like Lemma 3.2 ,

3.3. Infinite Cyclic Covers of 3-Manifolds. In light of equation (2), the matrix $B_{\infty}(\varphi)$ defines a map $D_{\infty} \rightarrow C_{\infty}$ which describes part of the action of $\tilde{\varphi}_{*}$. Its importance stems from the following immediate consequence of Proposition 2.5.

Proposition 3.5. Let $\varphi \in H S\left(a_{g}, b_{g}\right)$ and let $\left(N_{\varphi}\right)_{\infty} \rightarrow N_{\varphi}$ be the cover of $N_{\varphi}$ induced by $S_{\infty} \rightarrow S$. Then we have, as $\mathbb{Z}[\mathbb{Z}]-$ modules

$$
H_{1}\left(\left(N_{\varphi}\right)_{\infty} ; \mathbb{Z}\right)=C_{\infty} / \operatorname{im} B_{\infty}(\varphi)
$$

Thus $B_{\infty}(\varphi)$ is a presentation matrix of the $\mathbb{Z}[\mathbb{Z}]-$ module $H_{1}\left(\left(N_{\varphi}\right)_{\infty} ; \mathbb{Z}\right)$.

3.4. Finite Cyclic Covers. As we will explain in detail in Section 6, exponential torsion growth in a tower of cyclic covers of the manifold $N_{\varphi}$ will be governed by the logarithmic Mahler measure of the polynomial $\operatorname{det} B_{\infty}(\varphi)$. In Section 5 we will describe a criterion for positivity of this Mahler measure which is detectable in finite sub-covers of $S_{\infty}$. In this subsection we explain how $B_{\infty}(\varphi)$ affects the action on homology of lifts to finite sub-covers.

Choose $q \in \mathbb{N}_{\geq 3}$ and let $S_{q}$ be the $q$-fold cyclic cover of $S$ defined by algebraic intersection number with $a_{g} \bmod q$; denote by $G \cong \mathbb{Z} / q \mathbb{Z}$ its deck group. We want to describe the homology of $S_{q}$ as a $\mathbb{Z}[G]$-module (compare [Lo97, Section 4] for a very similar discussion) and its relation to the homology of $S_{\infty}$. 
The Chevalley-Weil theorem CW34 states that as a G-representation space, we have

$$
H_{1}\left(S_{q} ; \mathbb{C}\right) \cong \mathbb{C}[G]^{2 g-2} \oplus \mathbb{C}^{2} .
$$

In the case of cyclic covers, we can obtain this more explicitly (and with integral coefficients) as follows: $S_{q}$ is covered by $S_{\infty}$; denote by $\hat{\alpha}_{i}, \hat{\beta}_{i}, i<g$ the images of $\widetilde{\alpha}_{i}, \widetilde{\beta}_{i}$.

With an argument as in Lemma 3.1 one easily sees that the homology classes $\hat{a}_{i}, \hat{b}_{i}, 1 \leq i<g$ of the curves $\hat{\alpha}_{i}, \hat{\beta}_{i}$ span a free $\mathbb{Z}[G]$-submodule $\mathbb{Z}[G]^{2 g-2}$ of $H_{1}\left(S_{q} ; \mathbb{Z}\right)$. Choose lifts $\hat{\alpha}_{g}$ of $\alpha_{g}$ and $\hat{\beta}_{g}$ of $\beta_{g}$. It is easy to see that their homology classes $\hat{a}_{g}, \hat{b}_{g}$ span a trivial $\mathbb{Z}[G]$-module $\mathbb{Z}^{2}$.

Thus,

$$
F_{q}=\operatorname{span}_{\mathbb{Z}[G]}\left\{\hat{a}_{i}, \hat{b}_{i}, i<g\right\}
$$

is a free $\mathbb{Z}[G]$-submodule of $H_{1}\left(S_{q} ; \mathbb{Z}\right)$ which decomposes as

$$
F_{q}=C_{q} \oplus D_{q}, \quad D_{q}=\operatorname{span}_{\mathbb{Z}[G]}\left\{\hat{a}_{i}, i<g\right\}, C_{q}=\operatorname{span}_{\mathbb{Z}[G]}\left\{\hat{b}_{i}, i<g\right\}
$$

As in Lemma [3.2, any lift $\hat{\varphi}$ of an element $\varphi \in H S\left(a_{g}, b_{g}\right)$ respects the decomposition $H_{1}\left(S_{q} ; \mathbb{Z}\right) \cong F_{q} \oplus \mathbb{Z}^{2}$. Hence, we may define matrices $M_{q}(\varphi), B_{q}(\varphi)$ as in Definition 3.3 (replacing all lifts $\sim$ to $S_{\infty}$ by the corresponding lifts $\hat{\cdot}$ to $\left.S_{q}\right)$.

Summarising, we have the following.

Lemma 3.6. With the identifications as above, the covering map $S_{\infty} \rightarrow S_{q}$ induces on homology a map

$$
H_{1}\left(S_{\infty} ; \mathbb{Z}\right) \cong \mathbb{Z}[\mathbb{Z}]^{2 g-2} \oplus \mathbb{Z} \rightarrow \mathbb{Z}[G]^{2 g-2} \oplus \mathbb{Z}^{2} \cong H_{1}\left(S_{q} ; \mathbb{Z}\right)
$$

inducing via the quotient homomorphism $\mathbb{Z} \rightarrow \mathbb{Z} / q \mathbb{Z}$ a map between the free modules

$$
F_{\infty}=C_{\infty} \oplus D_{\infty} \rightarrow C_{q} \oplus D_{q}=F_{q}
$$

which respects the direct sum decompositions. Furthermore, $M_{q}(\varphi), B_{q}(\varphi)$ are the images of $M_{\infty}(\varphi), B_{\infty}(\varphi)$ under coordinate-wise application of the ring morphism $\mathbb{Z}[\mathbb{Z}] \rightarrow \mathbb{Z}[G]$ induced by $\mathbb{Z} \rightarrow \mathbb{Z} / q \mathbb{Z}$.

\section{The Torelli RePresentation for A Finite COVER}

The purpose of this section is to show that for a finite cover as discussed in Section 3.4, the matrices $M_{q}(\varphi), \varphi \in \mathcal{I}_{\mathrm{g}}$ map to a Zariski dense subset of some suitable algebraic group. This will be the main ingredient in Section 6 that allows to use the results by Benoist and Quint on random walks. We can restrict here to the case of $\varphi \in \mathcal{I}_{\mathrm{g}}$ for simplicity; as $\mathcal{I}_{\mathrm{g}}<H S\left(a_{g}, b_{g}\right)$ the image of $H S\left(a_{g}, b_{g}\right)$ will then also be Zariski dense.

The arguments rest on the results in [Lo97, Section 4]. We begin with explaining these results in the form we need. 
4.1. Skew-Hermitian modules. We require a few classical results on automorphism groups of skew-Hermitian modules; we only summarise the most important points here, details can be found in HO89.

Let $\mathcal{R}$ be a ring with an involution ${ }^{-}: \mathcal{R} \rightarrow \mathcal{R}$ which is a homomorphism with respect to the additive structure of $\mathcal{R}$. Let $\mathcal{M}$ be a module over $\mathcal{R}$. A skew-Hermitian form on $\mathcal{M}$ is a non-degenerate sesqui-linear form $\langle\cdot, \cdot\rangle$ : $\mathcal{M} \times \mathcal{M} \rightarrow \mathcal{R}$ such that

$$
\langle x, y\rangle=-\overline{\langle y, x\rangle}
$$

for all $x, y \in \mathcal{M}$.

Given a module $(\mathcal{M},\langle\rangle$,$) with such a form, we will denote the group of$ automorphisms that preserve the form by

$$
\mathrm{U}(\mathcal{M})=\{\alpha \in \operatorname{Aut}(\mathcal{M}) \mid\langle\alpha x, \alpha y\rangle=\langle x, y\rangle\} .
$$

It is well known that in the special case where $\mathcal{M}=\mathbb{C}^{2 n}$ for some $n \in \mathbb{N}$ and the involution is given by complex conjugation, we can find a basis

$$
\left\{x_{i}, y_{i}\right\}_{i=1}^{n}
$$

of $\mathbb{C}^{2 n}$ such that

$$
\left\langle x_{i}, y_{j}\right\rangle=\delta_{i j} \text { and }\left\langle x_{i}, x_{j}\right\rangle=\left\langle y_{i}, y_{j}\right\rangle=0
$$

for all $1 \leq i, j \leq n$. In this case we will write

$$
\mathrm{U}(\mathcal{M})=\mathrm{U}(n, n) .
$$

The usual symplectic group $\operatorname{Sp}_{2 n}(\mathbb{R})$ is precisely the subgroup of $\mathrm{U}(n, n)$ of all those matrices with real entries. We will write $\mathrm{SU}(n, n)$ for the subgroup of $U(n, n)$ of matrices with determinant 1 .

4.2. The representation. We are now ready to revisit Looijenga's arguments from [Lo97, Section 4] to study the set of matrices $M_{q}(\varphi)$ for $\varphi \in \mathcal{I}_{\mathrm{g}}$ (compare Section 3.4). We need the following set up.

Fix an integer $q \geq 3$ and let $G$ be a cyclic group of order $q$. Denote by $k=\mathbb{Q}\left[\zeta_{q}\right]$ the extension of $\mathbb{Q}$ by the $q$-th roots of unity. We identify $k$ with a subfield of the complex numbers. There is a map

$$
\iota: \mathbb{Z}[G] \rightarrow k \subset \mathbb{C}
$$

mapping a generator of $G$ to a primitive $q$-th root of unity 5 . We often consider the image of $\iota$ as contained in the complex numbers rather than in the abstract field $k$. Note that $\iota$ is not unique. Different choices of $\iota$ differ by an element of the Galois group of the field $k$. If $q$ is prime then $\iota$ can be chosen to send any given generator $1 \in G$ to any prescribed $q$-th root of unity. This freedom of choice will be important later (in Section 6). Until then, all arguments will work for any choice of $\iota$.

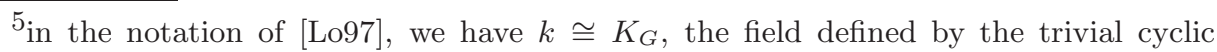
quotient of $G$
} 
Under $\iota$, the involution on $\mathbb{Z}[G]$ induced by $\bar{g}=g^{-1}$ corresponds to complex conjugation:

$$
\iota(\bar{p})=\overline{\iota(p)}, \quad \forall p \in \mathbb{Z}[G] .
$$

If $x \in \mathbb{Z}[G]^{n}$, we denote by $\iota x \in \mathbb{C}^{n}$ the vector obtained by applying $\iota$ coordinate-wise.

As explained in Section 3, we may assume without loss of generality that $S_{q} \rightarrow S$ is the regular cyclic cover of degree $q$ with deck group $G$ induced by algebraic intersection number $\bmod q$ with the curve $\alpha_{g}$. We use the notation from that section.

The homology $H_{1}\left(S_{q} ; \mathbb{Z}\right)$ is equipped with the usual intersection form $(\cdot, \cdot)$ as well as with a skew-Hermitian form $\Phi: H_{1}\left(S_{q} ; \mathbb{Z}\right) \times H_{1}\left(S_{q} ; \mathbb{Z}\right) \rightarrow \mathbb{Z}[G]$ (sometimes called Reidemeister pairing) given by

$$
\Phi(x, y)=\sum_{g \in G}(x, g y) g .
$$

Because $G$ preserves the submodule $F_{q} \subset H_{1}\left(S_{q} ; \mathbb{Z}\right)$, the bilinear form $\Phi$ defines a skew-Hermitian form on $F_{q} \cong \mathbb{Z}[G]^{2 g-2}$.

Furthermore, we have for all $x, y \in \mathbb{Z}[G]^{2 g-2}$ and $\varphi \in \mathcal{I}_{\mathrm{g}}$

$$
\Phi\left(M_{q}(\varphi) x, M_{q}(\varphi) y\right)=\Phi(x, y)
$$

since each lift of $\varphi$ preserves the intersection form $(\cdot, \cdot)$ and commutes with the deck group action.

Recall from Subsection 3.4 the definition of the classes $\hat{a}_{i}, \hat{b}_{k} \in H_{1}\left(S_{q} ; \mathbb{Z}\right)$. In what follows, we choose a basis $\left\{x_{i}, y_{i}\right\}_{i=1}^{g-1}$ for $\mathbb{C}^{2 g-2}$ such that:

$$
\iota\left(\hat{a_{i}}\right)=x_{i} \text { and } \iota\left(\hat{b_{i}}\right)=y_{i}
$$

for all $i=1, \ldots, g-1$. We also define a skew-Hermitian form $\langle\cdot, \cdot\rangle$ on $\mathbb{C}^{2 g-2}$ by

$$
\left\langle x_{i}, y_{i}\right\rangle=\delta_{i j} \text { and }\left\langle x_{i}, x_{j}\right\rangle=\left\langle y_{i}, y_{j}\right\rangle=0
$$

for all $i, j=1, \ldots, g-1$.

We now have the following

Lemma 4.1. For all $x, y \in H_{1}\left(S_{q} ; \mathbb{Z}\right)$ :

$$
\iota \Phi(x, y)=\langle\iota x, \iota y\rangle
$$

Proof. We have:

$$
\Phi\left(\hat{a}_{i}, \hat{b}_{j}\right)=\delta_{i j} \text { and }\left(\hat{a}_{i}, \hat{a}_{j}\right)=\left(\hat{b}_{i}, \hat{b}_{j}\right)=0
$$

The form $\Phi$ is skew-Hermitian and $\iota$ is linear by definition, hence the lemma.

By Lemma 4.1 and equation (4), for all $\varphi \in \mathcal{I}_{\mathrm{g}}$ the matrix $\iota M_{q}(\varphi)$ is contained in the group $\mathrm{U}(g-1, g-1)$ of skew Hermitian automorphisms of $\mathbb{C}^{2 g-2}$.

The assignment $\varphi \mapsto \iota M_{q}(\varphi)$ is not a representation of $\mathcal{I}_{\mathrm{g}}$ since the matrix $M_{q}(\varphi)$ depends on a choice of a lift of $\varphi$ to our cyclic cover, and therefore 
is defined only up to a unit in $\mathbb{Z}[G]$ (compare Section [3). The (complex) matrix $\iota M_{q}(\varphi)$ is thus also only defined up to multiplication by a $q$-th root of unity.

To avoid these problems, let $\widehat{\mathcal{I}}_{g}^{(q)}$ be the group of all lifts to $S_{q}$ of all elements of $\mathcal{I}_{\mathrm{g}}$. We then have a short exact sequence

$$
1 \rightarrow G \rightarrow \widehat{\mathcal{I}}_{g}^{(q)} \rightarrow \mathcal{I}_{\mathrm{g}} \rightarrow 1
$$

and an actual representation

$$
\rho_{q}: \widehat{\mathcal{I}}_{g}^{(q)} \rightarrow \mathrm{U}(g-1, g-1) .
$$

In Lo97, Looijenga studied this representation. More generally, denote by $\operatorname{Mod}(S)^{(q)}$ the finite index subgroup of $\operatorname{Mod}(S)$ of all elements which admit a lift to $S_{q}$. The group $\mathcal{I}_{\mathrm{g}}$ is a normal subgroup of $\operatorname{Mod}(S)^{(q)}$. As before, there is an exact sequence

$$
1 \rightarrow G \rightarrow \widehat{\operatorname{Mod}}(S)^{(q)} \rightarrow \operatorname{Mod}(S)^{(q)} \rightarrow 1 .
$$

Using the action on homology, we obtain a representation $\hat{\rho}_{q}: \widehat{\operatorname{Mod}}(S)^{(q)} \rightarrow$ $\mathrm{U}(g-1, g-1)$ extending the representation $\rho_{q}$.

Denote by $\mathrm{U}^{\sharp}(g-1, g-1)$ the subgroup of $\mathrm{U}(g-1, g-1)$ consisting of matrices whose determinant is equal to a square of a $q$-th root of unity. Note that $\mathrm{SU}(g-1, g-1)$ is a finite index subgroup of $\mathrm{U}^{\sharp}(g-1, g-1)$. Theorem 2.4 of [Lo97] states the following.

Theorem 4.2. Let $g \geq 3, q \geq 3$ and let $R_{q}$ be the ring of integers in the number field $\mathbb{Q}\left[\zeta_{q}\right]$. Then

$$
\hat{\rho}_{q}(\widehat{\operatorname{Mod}}(S))=U^{\sharp}\left(g-1, g-1 ; R_{q}\right) .
$$

Here the group $U^{\sharp}\left(g-1, g-1 ; R_{q}\right)$ is just the subgroup of $U^{\sharp}(g-1, g-1)$ of matrices with coefficients in $R_{q}$.

4.3. Denseness. We are now ready to prove that the image of the representation $\rho_{q}$ defined above is Zariski dense in $\mathrm{SU}(g-1, g-1)$.

The proof of the following proposition was suggested to us by an anonymous referee and replaces an earlier argument which followed Looijenga's paper [Lo97.

Proposition 4.3. Let $g \geq 3$ and $q \geq 3$; then

$$
\rho_{q}\left(\widehat{\mathcal{I}}_{g}^{(q)}\right) \cap \mathrm{SU}(g-1, g-1)
$$

is a finite index subgroup of $\mathrm{SU}\left(g-1, g-1 ; R_{q}\right)$.

Remark 4.4. The proposition is also true for $g=2$ and $q=5$ or $q \geq 7$ (compare [Lo97] and the proof below). Since we only need the $g \geq 3$ case, we do not give details. 
Proof. By Theorem 4.2, the image of the group $\widehat{\operatorname{Mod}}(S)^{(q)}$ under the representation $\hat{\rho}_{q}$ contains the group $\mathrm{SU}\left(g-1, g-1 ; R_{q}\right)$. Now for $g \geq 3$, the group $\mathrm{SU}\left(g-1, g-1 ; R_{q}\right)$ is arithmetic and of $\mathbb{Q}$-rank at least two. Furthermore, it contains the group

$$
A=\rho_{q}\left(\widehat{\mathcal{I}}_{\mathrm{g}}^{(q)}\right) \cap \mathrm{SU}\left(g-1, g-1 ; R_{q}\right)
$$

as a normal subgroup. Thus by Margulis's normal subgroup theorem, either $A$ is finite, or it has finite index. Thus we have to show that the group $A$ is infinite.

Write $\mathcal{M}=\hat{\rho}_{q}^{-1}\left(\mathrm{SU}\left(g-1, g-1 ; R_{q}\right)\right)$; since $U^{\sharp}\left(g-1, g-1 ; R_{q}\right)$ is a finite central extension of $\mathrm{SU}\left(g-1, g-1 ; R_{q}\right), \mathcal{M}$ is a finite index normal subgroup of $\widehat{\operatorname{Mod}}(S)^{(q)}$ which intersect the Torelli group $\hat{\mathcal{I}}_{\mathrm{g}}{ }^{(q)}$ in a finite index subgroup $\mathcal{P}$.

Let us denote by $\Pi: \mathcal{M} \rightarrow \operatorname{Sp}(2 g, \mathbb{Z})$ the natural projection. As $\mathcal{M}$ is a subgroup of $\operatorname{Mod}(S)$ of finite index, $\Pi(\mathcal{M})=\mathcal{H}$ has finite index in $\operatorname{Sp}(2 g, \mathbb{Z})$ and hence is a lattice of the simple Lie group $\operatorname{Sp}(2 g, \mathbb{R})$ of rank $g \geq 3$. Taking the quotient of $\mathcal{M}$ by the kernel $\mathcal{K}<\mathcal{M}$ of the homomorphism $\hat{\rho}$ gives rise to an exact sequence

$$
1 \rightarrow \mathcal{P} / \mathcal{K} \cap \mathcal{P} \rightarrow \mathcal{M} / \mathcal{K} \rightarrow \mathcal{H} / \Pi(\mathcal{K}) \rightarrow 1
$$

where the group $\mathcal{M} / \mathcal{K}$ is isomorphic to $\mathrm{SU}\left(g-1, g-1 ; R_{q}\right)$.

Now if the image of the group $\widehat{\mathcal{I}}_{\mathrm{g}}^{(q)}$ under the representation $\rho_{q}$ is finite then $\mathcal{P} / \mathcal{K}$ is a finite group. Thus this sequence describes the arithmetic group $\mathrm{SU}\left(g-1, g-1 ; R_{q}\right)$ as an extension of the group $\mathcal{H} / \Pi(\mathcal{K})$ by a finite group. This implies that $\mathcal{H} / \Pi(\mathcal{K})$ is infinite. Margulis's normal subgroup theorem now shows that $\Pi(\mathcal{K})$ is a finite normal subgroup of the higher rank lattice $\mathcal{H}$.

Now $\mathcal{H}<\operatorname{Sp}(2 g, \mathbb{R})$ is Zariski dense and therefore a finite normal subgroup of $\mathcal{H}$ is normalized by the entire simple group $\operatorname{Sp}(2 g, \mathbb{R})$. Then the group has to be central in $\operatorname{Sp}(2 g, \mathbb{R})$ and hence either it is trivial, or it equals \pm 1 . As a consequence, the group $\mathcal{H} / \Pi(\mathcal{K})$ either equals a lattice in $\operatorname{Sp}(2 g, \mathbb{R})$, or a lattice in the quotient $\mathrm{P} \operatorname{Sp}(2 g, \mathbb{R})$ of this group by its center.

To summarize, under the assumption that $\rho_{g}(\mathcal{P})$ is not a subgroup of $\mathrm{SU}\left(g-1, g-1 ; R_{q}\right)$ of finite index we conclude that the arithmetic group $\mathrm{SU}\left(g-1, g-1 ; R_{q}\right)$ is a finite extension of a lattice in $\mathrm{P} \operatorname{Sp}(2 g, \mathbb{R})$. But $\mathrm{SU}\left(g-1, g-1 ; R_{q}\right)$ is an irreducible lattice in a higher rank semi-simple Lie group $G$ which does not contain any factor locally isomorphic to the higher rank simple Lie group $\operatorname{Sp}(2 g, \mathbb{R})$. This contradicts Margulis' super-rigidity theorem for lattices.

Remark 4.5. Let $\varepsilon: \mathbb{Q}\left[\zeta_{q}\right] \rightarrow \mathbb{Z}$ be the $\mathbb{Z}$-linear augmentation map defined by $\varepsilon\left(\zeta_{q}\right)=1$ for ever $q$-th root of unity $\zeta_{q}$. It follows from the proof of Proposition 4.3 that the image of $\mathcal{I}_{\mathrm{g}}$ under $\rho_{q}$ intersects $\mathrm{SU}\left(g-1, g-1 ; R_{p}\right)$ in the finite index normal subgroup defined as the preimage of the identity 
under the map which associates to a matrix its coordinate-wise image under $\varepsilon$. We do not know whether the image coincides with this group.

In the following corollary, we view $\mathrm{SU}\left(g-1, g-1 ; R_{q}\right)$ as a countable subgroup of the Lie group $\mathrm{SU}(g-1, g-1)$ (and not as an arithmetic group).

Corollary 4.6. For $g \geq 3, q \geq 3$ the group $\rho_{q}\left(\widehat{\mathcal{I}}_{g}^{(q)}\right) \cap \mathrm{SU}(g-1, g-1)$ is Zariski dense in $\mathrm{SU}(g-1, g-1)$.

Proof. By Proposition 4.3 , if the degree over $\mathbb{Q}$ of the number field $\mathbb{Q}\left[\zeta_{q}\right]$ is at least four then $\rho_{q}\left(\widehat{\mathcal{I}}_{g}^{(q)}\right) \cap \mathrm{SU}(g-1, g-1)$ is a lattice in an algebraic group which has $\mathrm{SU}(g-1, g-1)$ as a factor. In this case the image group is dense in the usual topology.

Otherwise, as $q \geq 3$, the degree over $\mathbb{Q}$ of $\mathbb{Q}\left[\zeta_{q}\right]$ is two and $\rho_{q}\left(\widehat{\mathcal{I}}_{g}^{(q)}\right) \cap$ $\mathrm{SU}(g-1, g-1)$ is a lattice in $\mathrm{SU}(g-1, g-1)$ and hence Zariski dense as well.

4.4. The action on subspaces: Part I. The core ingredient in the proof of the main theorem will be to control the determinant of $B_{q}(\varphi)$ (introduced in Section 3). This will be done using random walks on algebraic groups in Section 6.1. In this section we describe how to detect this determinant, using the action of $\mathrm{U}(g-1, g-1)$ on subspaces of $\mathbb{C}^{2 g-2}$.

Recall that the exterior product of a basis of a $(g-1)$-dimensional subspace of $\mathbb{C}^{2 g-2}$ is a pure vector in $\wedge^{g-1} \mathbb{C}^{2 g-2}$. Thus the action of $\mathrm{U}(g-$ $1, g-1)$ on the Grassmannian of half-dimensional subspaces of $\mathbb{C}^{2 g-2}$ is encoded in the natural representation of $\mathrm{U}(g-1, g-1)$ on $\wedge^{g-1} \mathbb{C}^{2 g-2}$. This representation is irreducible (see Section 6.3).

Let $\left\{x_{i}, y_{i}\right\}_{i=1}^{g-1}$ denote a basis of $\mathbb{C}^{2 g-2}$ with respect to which $\langle\cdot, \cdot\rangle$ takes the standard form (see Subsection 4.2). Let $e, f \in \wedge^{g-1} \mathbb{C}^{2 g-2}$ be defined by

$$
e=x_{1} \wedge \cdots \wedge x_{g-1} \text { and } f=y_{1} \wedge \cdots \wedge y_{g-1} .
$$

Recall that we want to control the lower left block in the image of our random element of $\widehat{\mathcal{I}}_{g}^{(q)}$ under $\rho_{q}$. The first observation is that this determinant appears as the $f$-coefficient of $\rho_{q}(\hat{\varphi}) e$. To this end, let $(\cdot, \cdot)$ denote the Hermitian inner product on $\wedge^{g-1} \mathbb{C}^{2 g-2}$ corresponding to the Hermitian inner product on $\mathbb{C}^{2 g-2}$ for which the basis $\left\{x_{i}, y_{i}\right\}_{i=1}^{g-1}$ is orthonormal.

Lemma 4.7. Let $\varphi \in \mathcal{I}_{\mathrm{g}}$ be arbitrary, and let $\hat{\varphi} \in \widehat{\mathcal{I}}_{g}^{(q)}$ be any lift of $\varphi$. Then

$$
\left|\left(\rho_{q}(\hat{\varphi}) e, f\right)\right|=\left|\iota \operatorname{det}\left(B_{q}(\varphi)\right)\right| .
$$

Proof. First note that a lift $\hat{\varphi}$ is defined up to multiplication by a deck group element, which under $\iota$ maps to multiplication by a complex number of absolute value 1 . Thus both sides of the inequality are independent of the choice of lift. Similarly, we can choose our preferred lift of $\varphi$ to compute $\iota B_{q}(\varphi)$. 
Let

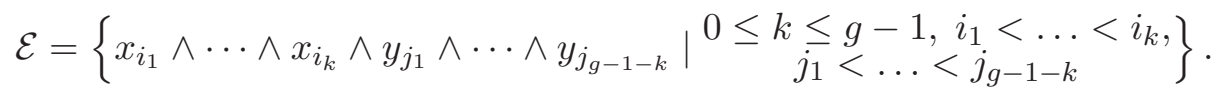

$\mathcal{E}$ is a orthonormal basis for $\wedge^{g-1} \mathbb{C}^{2 g-2}$. Because $f$ is orthogonal to all elements of $\mathcal{E}$ not equal to it, we obtain

$$
\begin{aligned}
\left(\rho_{q}(\varphi) e, f\right) & =\sum_{i_{1}, \ldots, i_{g-1}=1}^{g-1}\left(\left(B_{q}(\varphi)\right)_{1, i_{1}} y_{i_{1}} \wedge \ldots \wedge\left(B_{q}(\varphi)\right)_{g-1, i_{g-1}} y_{i_{g-1}}, f\right) \\
& =\sum_{i_{1}, \ldots, i_{g-1}=1}^{g-1}\left(y_{i_{1}} \wedge \ldots \wedge y_{i_{g-1}}, f\right) \prod_{j=1}^{g-1}\left(B_{q}(\varphi)\right)_{j, i_{j}} .
\end{aligned}
$$

The inner products in the terms satisfy

$$
\left(y_{i_{1}} \wedge \ldots \wedge y_{i_{g-1}}, f\right)= \begin{cases}\varepsilon\left(i_{1}, \ldots, i_{g-1}\right) & \text { if the map } j \mapsto i_{j} \text { is bijective } \\ 0 & \text { otherwise }\end{cases}
$$

where $\varepsilon\left(i_{1}, \ldots, i_{g-1}\right)$ denotes the sign of the map $j \mapsto i_{j}$ viewed as a permutation. As such, we can view the sum above as a sum over elements of the symmetric group on $g-1$ letters $\mathfrak{S}_{g-1}$. We obtain:

$$
\left(\rho_{q}(\varphi) e, f\right)=\sum_{\pi \in \mathfrak{S}_{g-1}} \varepsilon(\pi) \prod_{j=1}^{g-1}\left(B_{q}(\varphi)\right)_{j, \pi(j)},
$$

where $\varepsilon(\pi)$ denotes the sign of a permutation $\pi \in \mathfrak{S}_{g-1}$. The expression above is the Leibniz formula for the determinant of $B_{q}(\varphi)$.

\section{Mahler measures}

In this section we review some basic properties of the logarithmic Mahler measure of polynomials and establish conditions which are satisfied in the case that the polynomials $\operatorname{det} B_{\infty}(\varphi)$ from Section 3 have logarithmic Mahler measure zero.

5.1. Mahler measure obstructions. First, we collect some classical facts on integral polynomials in a single variable and their Mahler measures.

Let $P$ be a polynomial in one variable with integral coefficients. The (logarithmic) Mahler measure of $P$ is defined as

$$
m(P)=\frac{1}{2 \pi} \int_{0}^{2 \pi} \ln (|P(\exp (i \theta))|) d \theta .
$$

Note that $m\left(t^{k} P\right)=m(P)$ for all $k \in \mathbb{Z}$.

We let $M(P)=\exp (m(P))$ be the multiplicative Mahler measure of $P$. If we speak simply of Mahler measure, we will usually mean the logarithmic Mahler measure. If we write $P(t)=a \prod_{i}\left(t-\alpha_{i}\right)$ with $\alpha_{i} \in \mathbb{C}$ then by Jensen's formula, we have

$$
M(P)=|a| \prod \max \left(1,\left|\alpha_{i}\right|\right)
$$


For $n \in \mathbb{N}$, the $n^{\text {th }}$ cyclotomic polynomial $\Phi_{n}$ in $t$ is the unique irreducible polynomial (over $\mathbb{Z}$ ) that is a divisor of $t^{n}-1$ but not of $t^{k}-1$ for any $k<n$, $k \in \mathbb{N}$. As such, its roots are exactly the $n^{\text {th }}$ primitive roots of unity. This in turn implies that

$$
\operatorname{deg}\left(\Phi_{n}\right)=\varphi(n)
$$

for all $n \in \mathbb{N}$, where $\varphi: \mathbb{N} \rightarrow \mathbb{N}$ denotes Euler's totient function.

The following proposition is a well-known application of a result of Kronecker [Kr57] (compare e.g. [EW, Theorem 1.31]).

Proposition 5.1. Let $P(t)$ be a polynomial in one variable with integral coefficients. Then $m(P(t)) \geq 0$, with equality if and only if $P(t)$ is a product of cyclotomic polynomials and powers of $t$.

From the proposition above we will derive a condition on polynomials of Mahler measure zero. Before we can prove it, we need two classical results on cyclotomic polynomials and Euler's totient function. To this end, let $\mathrm{c}\left(\Phi_{m}\right)$ denote the maximum of the absolute values of the coefficients of $\Phi_{m}$. The following bound on $\mathrm{c}\left(\Phi_{m}\right)$ is due to Maier.

Proposition 5.2. Ma96] Let $\eta: \mathbb{N} \rightarrow \mathbb{R}$ be a function so that

$$
\eta(n) \rightarrow \infty
$$

as $n \rightarrow \infty$. Then for all but finitely many $m \in \mathbb{N}$ we have

$$
\mathrm{c}\left(\Phi_{m}\right) \leq m^{\eta(m)} .
$$

Of course, the set of $m \in \mathbb{N}$ for which the above bound above does not hold depends on the choice of function $\eta$.

Finally, we need the following bound for the totient function (see e.g. [HW08, Theorem 327]). In fact, sharper estimates on $\varphi(n)$ are available, but the following is enough for our purpose.

Proposition 5.3. For every $\delta \in(0,1)$ we have:

$$
\frac{\varphi(n)}{n^{\delta}} \rightarrow \infty
$$

as $n \rightarrow \infty$.

We are now ready to prove our condition on polynomials.

Proposition 5.4. For every $\alpha>0$ there exists a finite set $K_{\alpha} \subset \mathbb{N}$ with the following property. If $P \in \mathbb{Z}[t]$ satisfies $m(P)=0$ then

- either $\Phi_{k}$ is a factor of $P$ for some $k \in K_{\alpha}$,

- or for all $\xi \in \mathbb{C}$ with $|\xi|=1$ we have

$$
|P(\xi)| \leq \exp (\alpha \operatorname{deg}(P)) .
$$

Proof. Proposition 5.3 (applied to $\delta=1 / 2$ ) allows us to find some $n_{0} \in \mathbb{N}$ so that

$$
\varphi(n)>\sqrt{n},
$$


for all $n \geq n_{0}$. Define $\eta: \mathbb{N} \rightarrow \mathbb{R}$ by

$$
\eta(n)=\alpha \cdot \frac{\sqrt{n}}{\log (n)}-1
$$

Using Proposition 5.2, we obtain a finite set $K^{\prime} \subset \mathbb{N}$ so that the coefficients of all $\Phi_{m}$ for $m \notin K^{\prime}$ are bounded in absolute value by $m^{\eta(m)}$. This implies that for $m \notin K^{\prime}$ we have

$$
\left|\Phi_{m}(\xi)\right| \leq \varphi(m) \cdot m^{\eta(m)}
$$

for all $\xi \in \mathbb{C}$ with $|\xi|=1$. The same holds trivially for the polynomial $P(t)=t$.

Define $K_{\alpha} \subset \mathbb{N}$ by

$$
K_{\alpha}=K^{\prime} \cup\left\{0,1, \ldots, n_{0}\right\} .
$$

We claim that the proposition holds for this set. As such, we need to check the inequality in the statement of the proposition for all polynomials of Mahler measure zero not containing any factor $\Phi_{k}$ for some $k \in K_{\alpha}$.

Let $P \in \mathbb{Z}[t]$ a polynomial with $m(P)=0$. Proposition 5.1 tells us that we may write

$$
P(t)=t^{k} \prod_{i=1}^{r} \Phi_{m_{i}}(t)
$$

for some $m \in \mathbb{N}^{r}$ and $k, r \in \mathbb{N}$. Assume that $m_{i} \notin K_{\alpha}$ for all $i=1, \ldots, r$. We have

$$
\operatorname{deg}(P)=k+\sum_{i=1}^{r} \varphi\left(m_{i}\right)>\sum_{i=1}^{r} \sqrt{m_{i}} .
$$

By the choice of $K_{\alpha}$, for all $\xi \in \mathbb{C}$ with $|\xi|=1$ we obtain that

$$
|P(\xi)| \leq \prod_{i=1}^{r} \varphi\left(m_{i}\right) m_{i}^{\alpha \sqrt{m_{i}} / \log \left(m_{i}\right)-1} \leq \prod_{i=1}^{r} m_{i}^{\alpha \cdot \sqrt{m_{i}} / \log \left(m_{i}\right)},
$$

where we have used that $\varphi(n) \leq n$ for all $n \in \mathbb{N}$. Hence

$$
|P(\xi)| \leq \exp \left(\alpha \sum_{i=1}^{r} \sqrt{m_{i}}\right) \leq \exp (\alpha \cdot \operatorname{deg}(P))
$$

\section{Finishing the PROOF}

In this section we finish the proof of our main theorems. We will again restrict to the case of random walks on the Torelli group. The case of the homology stabilisers is completely analogous.

Let $S$ be a surface of genus $g \geq 3$ and let $\sigma: \pi_{1}(S) \rightarrow \mathbb{Z}$ be a surjection as in Section 2 so that $\operatorname{ker}(\sigma) \supset K=\operatorname{ker}\left(\pi_{1}(S) \rightarrow \pi_{1}(V)\right)$. Let $S_{\infty}, S_{q}$ be the covers considered in Section 3 induced by $\sigma$. For $\varphi \in \mathcal{I}_{\text {g }}$ and $q \in \mathbb{N}$ let 
$\left(N_{\varphi}\right)_{q}$ denote the cover of $N_{\varphi}$ defined by the map $\pi_{1}\left(N_{\varphi}\right) \rightarrow \mathbb{Z} / q \mathbb{Z}$ which factors through $\sigma$. Say that $\varphi$ has property $\mathrm{E}_{\sigma}$ if

$$
\lim _{q \rightarrow \infty} \frac{\log \left|H_{1}\left(\left(N_{\varphi}\right)_{q} ; \mathbb{Z}\right)_{\text {tor }}\right|}{q}
$$

exists and is positive.

Our goal is to prove:

Theorem 6.1. Let $\mu$ be any finitely supported measure on the Torelli group $\mathcal{I}_{\mathrm{g}}<\operatorname{Mod}(S)$ whose support generates $\mathcal{I}_{\mathrm{g}}$ as a semigroup. Then we have

$$
\mu^{* n}\left(\left\{\varphi \in \mathcal{I}_{\mathrm{g}}, \varphi \text { has property } \mathrm{E}_{\sigma}\right\}\right) \rightarrow 1
$$

as $n \rightarrow \infty$.

By Proposition 3.5. $B_{\infty}(\varphi)$ is a presentation matrix for $H_{1}\left(\left(N_{\varphi}\right)_{\infty} ; \mathbb{Z}\right)$. We can now apply [Ra12, Theorem 3.1] (where $m=1, \varphi=\sigma, \hat{X}=\left(N_{\varphi}\right)_{\infty}, X_{q}=$ $\left.\left(N_{\varphi}\right)_{q}\right)$ and obtain the following.

Proposition 6.2. With notation as above, if $m\left(\operatorname{det} B_{\infty}(\varphi)\right)>0$ then

$$
\lim _{q \rightarrow \infty} \frac{\log \left|H_{1}\left(X_{q} ; \mathbb{Z}\right)_{\text {tor }}\right|}{q}=m\left(\operatorname{det} B_{\infty}(\varphi)\right)
$$

exists and is positive.

Thus, if $\varphi$ does not have $E_{\sigma}$, then we have that $m\left(\operatorname{det} B_{\infty}(\varphi)\right)=0$. The rest of this section will be devoted to showing that with probability converging to one, the logarithmic Mahler measure of $\left.\operatorname{det} B_{\infty}(\phi)\right)$ does not vanish. In order to be able to apply results on random walks on Lie groups later on, we first translate this into a condition on the image of (lifts of) $\varphi$ under the representations $\rho_{m}$ for some $m \in \mathbb{N}$.

6.1. Matrix Conditions. Recall from Lemma 4.7 that the determinant of $B_{q}(\varphi)$ can be computed from the action of $\rho_{q}(\hat{\varphi})$ on $\wedge^{g-1} \mathbb{C}^{2 g-2}$. Using the above, the condition in Proposition 5.4 translates as follows. Here, $e$ and $f$ are as in Section 4.4.

Proposition 6.3. Let $\alpha>0$ and let $\mu$ be a finitely supported probability measure on $\mathcal{I}_{\mathrm{g}}$ whose support generates $\mathcal{I}_{\mathrm{g}}$ as a semigroup. There exists a finite set $K_{\alpha, \mu} \subset \mathbb{N}$ such that the following holds.

Let $n$ be arbitrary and $\varphi \in \operatorname{supp}\left(\mu^{* n}\right) \subset \mathcal{I}_{\mathrm{g}}$ with $m\left(\operatorname{det} B_{\infty}(\varphi)\right)=0$. Then

- either $\Phi_{k}$ is a factor of $\operatorname{det} B_{\infty}(\varphi)$ for some $k \in K_{\alpha, \mu}$, and therefore $\left(\rho_{k}(\hat{\varphi}) e, f\right)=0$ for that $k$ and any lift $\hat{\varphi}$ of $\varphi$ to $S_{k}$,

- or for all $q \in \mathbb{N}$ we have

$$
\left|\left(\rho_{q}(\hat{\varphi}) e, f\right)\right| \leq \exp (\alpha \cdot n)
$$

for any lift $\hat{\varphi}$ of $\varphi$ to $S_{q}$. 
Proof. Let $S=\operatorname{supp}(\mu) \subset \mathcal{I}_{\text {g }}$ and let us choose lifts of all $s \in S$ so that the corresponding $M_{\infty}(s)$ are matrices whose coefficients are polynomials (as opposed to Laurent polynomials). Because $S$ is finite, this may be achieved by multiplying by some large power of $t$.

We will first derive a degree bound on $\operatorname{det}\left(B_{\infty}(\varphi)\right)$ for $\varphi \in \operatorname{supp}\left(\mu^{* n}\right)$. Let

$$
d_{\mu}=\max \left\{\operatorname{deg}\left(\left(M_{\infty}(s)\right)_{i j}\right) \mid i, j=1, \ldots, 2 g-2, s \in S\right\} .
$$

Any $M_{\infty}(\varphi)$ for $\varphi \in \operatorname{supp}\left(\mu^{* n}\right)$ is obtained by multiplying at most $n$ elements of $\left\{M_{\infty}(s) \mid s \in S\right\}$. Hence the coefficients of $M_{\infty}(\varphi)$ have degree at most $d_{\mu} \cdot n$. The polynomial $\operatorname{det}\left(B_{\infty}(\varphi)\right)$ is obtained by taking the determinant of a $(g-1) \times(g-1)$ block of $M_{\infty}(\varphi)$. As such, we obtain

$$
\operatorname{deg}\left(\operatorname{det}\left(B_{\infty}(\varphi)\right)\right) \leq(g-1) \cdot d_{\mu} \cdot n \text {. }
$$

Set $\alpha^{\prime}=\alpha /\left((g-1) d_{\mu}\right)$, and let $K_{\alpha^{\prime}} \subset \mathbb{N}$ be the finite set obtained from Proposition 5.4 applied with $\alpha^{\prime}$. We put $K_{\alpha, \mu}=K_{\alpha^{\prime}}$.

Now suppose that $\varphi$ is so that $m\left(\operatorname{det} B_{\infty}(\varphi)\right)=0$. Then by Proposition 5.4, either $\Phi_{k}$ divides $\operatorname{det}\left(B_{\infty}(\varphi)\right)$ for some $k \in K_{\alpha^{\prime}}$ or

$$
\left|\operatorname{det}\left(B_{\infty}(\varphi)\right)(\xi)\right| \leq \exp \left(\alpha^{\prime} \operatorname{deg}\left(\operatorname{det}\left(B_{\infty}(\varphi)\right)\right)\right)
$$

for all $\xi$ with $|\xi|=1$. In the first case, every primitive $k$-th root of unity is a zero of $\Phi_{k}$ and hence of $\operatorname{det}\left(B_{\infty}(\varphi)\right)$. Since $\iota \operatorname{det}\left(B_{k}(\varphi)\right)$ is obtained from $\operatorname{det}\left(B_{\infty}(\varphi)\right)$ by evaluating at a primitive $k$-th root of unity, this implies the claim (using Lemma 4.7).

In the latter case, the same argument yields that, for $\zeta_{q}$ a primitive $q$-th root of unity,

$$
\begin{gathered}
\left|\left(\rho_{q}(\hat{\varphi}) e, f\right)\right|=\left|\iota \operatorname{det}\left(B_{q}(\varphi)\right)\right|=\left|\operatorname{det}\left(B_{\infty}(\varphi)\right)\left(\zeta_{q}\right)\right| \\
\leq \exp \left(\alpha^{\prime} \operatorname{deg}\left(\operatorname{det}\left(B_{\infty}(\varphi)\right)\right)\right) \leq \exp (\alpha n) .
\end{gathered}
$$

6.2. Set-up and walks on $\widehat{\mathcal{I}}_{g}^{(q)}$. Let $q \geq 3$, and $\zeta_{q}$ be a primitive $q$-th root of unity. Then $k=\mathbb{Q}\left[\zeta_{q}\right]$ is the cyclotomic field of degree $\varphi(q)$ over $\mathbb{Q}$. Recall from Subsection 4.2 that we have a ring morphism

$$
\iota: \mathbb{Z}[G] \rightarrow k \subset \mathbb{C}
$$

where $G$ is the cyclic group with $q$ elements. It image is contained in the ring of integers of $k$. The morphism $\iota$ depends on the choice of $\zeta_{q}$.

By the results of Section 4 , the ring morphism $\iota$ induces a representation

$$
\rho_{q}: \widehat{\mathcal{I}}_{g}^{(q)} \rightarrow \mathrm{U}(g-1, g-1)
$$

whose image is contained in the subgroup $U^{\sharp}(g-1, g-1)$ of all elements with determinant a square of a $q$-th root of unity. Here as before, $\widehat{\mathcal{I}}_{g}^{(q)}$ is the group of all lifts of elements in the Torelli group to the surface $S_{q}$, and this group fits into the exact sequence

$$
1 \rightarrow G \rightarrow \widehat{\mathcal{I}}_{g}^{(q)} \rightarrow \mathcal{I}_{\mathrm{g}} \rightarrow 1
$$


In the statement of our main theorem, we use a random walk on the Torelli group $\mathcal{I}_{\mathrm{g}}$. To apply the representations $\rho_{q}$ and the criteria for Mahler measure 0 above, we have to work with random walks on a finite number of groups $\widehat{\mathcal{I}}_{g}^{(q)}$. The rest of this section is devoted to explaining how to pass from one to the other.

Let $\mu$ be a probability measure on $\mathcal{I}_{\mathrm{g}}$ whose support is finite and generates $\mathcal{I}_{\mathrm{g}}$ as a semigroup. Define a measure $\zeta$ on $\widehat{\mathcal{I}}_{g}^{(q)}$ via

$$
\zeta(h)=|G|^{-1} \mu(h G) .
$$

Then $\zeta$ is a measure whose finite support generates $\widehat{\mathcal{I}}_{g}^{(q)}$ as a semigroup.

Lemma 6.4. Let $\pi: \widehat{\mathcal{I}}_{g}^{(q)} \rightarrow \mathcal{I}_{\mathrm{g}}$ be the quotient map. Then $\zeta^{* n}\left(\pi^{-1} A\right)=$ $\mu^{* n}(A)$ for any $A \subset \mathcal{I}_{\mathrm{g}}$.

Proof. Let $Z \subset \widehat{\mathcal{I}}_{g}^{(q)}$ be a complete set of coset representatives for $\mathcal{I}_{\mathrm{g}}$ (under the quotient map $\pi$ ).

Then

$$
\begin{aligned}
& \zeta^{* n}\left(\pi^{-1} A\right)=\sum_{h_{1}, \ldots, h_{n} \in \widehat{\mathcal{I}}_{g}^{(q)}, h_{1} \cdots h_{n} \in \pi^{-1} A} \zeta\left(h_{1}\right) \cdots \zeta\left(h_{n}\right) \\
& =\sum_{a_{1}, \ldots, a_{n} \in Z, g_{1}, \ldots, g_{n} \in G, a_{1} \cdots a_{n} G \in A} \zeta\left(a_{1} g_{1}\right) \cdots \zeta\left(a_{n} g_{n}\right) \\
& =\sum_{a_{1}, \ldots, a_{n} \in Z, g_{1}, \ldots, g_{n} \in G, g_{1}, \ldots, g_{n} G \in A} \frac{\mu\left(a_{1} g_{1} G\right)}{|G|} \cdots \frac{\mu\left(a_{n} g_{n} G\right)}{|G|} \\
& =\sum_{a_{1}, \ldots, a_{n} \in Z, a_{1} \cdots a_{n} G \in A} \mu\left(a_{1} G\right) \cdots \mu\left(a_{n} G\right) \\
& =\sum_{l_{1}, \ldots, l_{k} \in \mathcal{I}_{\mathrm{g}}, l_{1} \cdots l_{n} \in A} \mu\left(l_{1}\right) \cdots \mu\left(l_{n}\right) \\
& =\mu^{* n}(A) \text {. }
\end{aligned}
$$

6.3. The action on subspaces: Part II. Before we can complete the proof, we need to collect some final facts on the representation $\rho_{q}: \widehat{\mathcal{I}}_{g}^{(q)} \rightarrow$ $U(g-1, g-1)$ and its action on subspaces. Recall that we are interested in the representation

$$
\sigma_{q}: \widehat{\mathcal{I}}_{g}^{(q)} \rightarrow \mathrm{GL}\left(\wedge^{g-1} \mathbb{C}^{2 g-2}\right)
$$

Recall that a representation $\rho$ of a group $G$ on a finite dimensional complex vector space $V$ is strongly irreducible if it is irreducible and if $\rho(G)$ does not preserve any finite union of proper subspaces.

The following proposition is standard.

Proposition 6.5. The representation of $\mathrm{SU}(g-1, g-1)$ on $\wedge^{g-1} \mathbb{C}^{2 g-2}$ induced by the standard action on $\mathbb{C}^{2 g-2}$ is strongly irreducible. The image of $\mathrm{SU}(g-1, g-1)$ in $\mathrm{GL}\left(\wedge^{g-1} \mathbb{C}^{2 g-2}\right)$ is semi-simple and Zariski closed. 
Proof. It is a classical fact that for any $k$, the representation of $\mathrm{SL}(d, \mathbb{C})$ on $\wedge^{k} \mathbb{C}^{d}$ is irreducible (compare e.g. [FH91, §15.2]). Now, $\mathrm{SU}(g-1, g-1)$ is a simple non-compact real Lie group with Lie algebra $\mathfrak{s u}_{g-1, g-1}$. We have that $\mathfrak{s u}_{g-1, g-1} \otimes \mathbb{C}=\mathfrak{s l}_{2 g-2}$. This implies that restrictions of irreducible representations of $\mathrm{SL}(2 g-2, \mathbb{C})$ to $\mathrm{SU}(g-1, g-1)$ are irreducible (compare the discussion in [FH91, §26.1, p. 439]). Since SU $(g-1, g-1)$ is connected, this representation is in fact strongly irreducible.

Now the map $\mathrm{SU}(g-1, g-1) \rightarrow \mathrm{GL}\left(\wedge^{g-1} \mathbb{C}^{2 g-2}\right)$ is algebraic, and images of algebraic groups under algebraic maps are Zariski closed. Furthermore, as $\mathrm{SU}(g-1, g-1)$ is simple, the same holds true for the image group.

Let $\Gamma$ be any countable group. A representation $\sigma: \Gamma \rightarrow \operatorname{GL}(V)$ on a complex vector space $V$ is called proximal if there exists a sequence $\left(g_{n}\right) \subset$ $\sigma(\Gamma) \subset \mathrm{GL}(V)$ and a sequence $\left(\lambda_{n}\right) \subset \mathbb{C}$ such that

$$
\pi=\lim _{n} \lambda_{n} g_{n}
$$

is an endomorphism of rank one.

Lemma 6.6. Let as before $\sigma_{q}: \widehat{\mathcal{I}}_{g}^{(q)} \rightarrow \mathrm{GL}\left(\wedge^{g-1} \mathbb{C}^{2 g-2}\right)$. Then the image of the representation $\sigma_{q}$ is strongly irreducible and proximal.

Proof. By Corollary 4.6 the image of $\rho_{q}$ intersects $\mathrm{SU}(g-1, g-1)$ in a Zariski dense subgroup. Thus, strong irreducibility of $\sigma_{q}$ follows from Proposition 6.5.

To show that $\sigma_{q}$ is proximal, it suffices to find an element $A$ in the image of $\widehat{\mathcal{I}}_{g}^{(q)}$ whose action on $\wedge^{g-1} \mathbb{C}^{2 g-2}$ is proximal. Now any matrix $A \in \mathrm{SU}(g-$ $1, g-1)$ as a $(2 g-2) \times(2 g-2)$-matrix with real eigenvalues $\lambda_{1}>\cdots>$ $\lambda_{g-1}>1>\lambda_{g-1}^{-1}>\cdots>\lambda_{1}^{-1}$ will do, and we can find such a matrix in the image of $\widehat{\mathcal{I}}_{g}^{(q)}$ since this image is Zariski dense in $\mathrm{SU}(g-1, g-1)$ by Corollary 4.6 again.

6.4. Random walks on $\widehat{\mathcal{I}}_{g}^{(q)}$.

In Proposition 6.3, for a choice of a positive number $\alpha>0$ we formulated two events for a random walk on $\mathcal{I}_{\mathrm{g}}$ and proved that at least one of these events occurs if the logarithmic Mahler measure of the Alexander polynomial of the hyperbolic three-manifold defined by an element of the walk vanishes. Our end game will be to find a number $\alpha$ so that the probability of any one of these two events occurring tends to zero with the step-length of the random walk.

To find such a number $\alpha>0$ we use results of Benoist and Quint BQ14, BQ16. Let us fix a finitely supported probability measure $\mu$ on $\widehat{\mathcal{I}}_{g}^{(q)}$ whose support generates $\widehat{\mathcal{I}}_{g}^{(q)}$ as a semigroup. Later, this measure will be obtained from a measure on the Torelli group $\mathcal{I}_{\mathrm{g}}$ using the procedure described in Section 6.2, but for this section this is not important. This set up gives rise to a one-sided Bernoulli space $B$ with alphabet $\widehat{\mathcal{I}}_{g}^{(q)}$ defined by:

$$
B=\left(\widehat{\mathcal{I}}_{g}^{(q)}\right)^{\mathbb{N}},
$$


The $\sigma$-algebra on this space is generated by cylinder sets

$$
C\left(\varphi_{1}, \ldots, \varphi_{n}\right)=\left\{b \in B \mid b_{i}=\varphi_{i} \forall i=1, \ldots, n\right\},
$$

for $\varphi_{1}, \ldots, \varphi_{n} \in \widehat{\mathcal{I}}_{g}^{(q)}$. We define a shift invariant probability measure $\beta$ on $B$ by

$$
\beta=\mu^{\otimes \mathbb{N} *} .
$$

Observe that by definition we have for a subset $X \subset \widehat{\mathcal{I}}_{g}^{(q)}$

$$
\mu^{* n}(X)=\beta\left(\bigcup_{\substack{\varphi_{1}, \ldots, \varphi_{n} \in \widehat{\mathcal{I}}_{g}^{(q)} \\ \varphi_{1} \cdots \varphi_{n} \in X}} C\left(\varphi_{1}, \ldots, \varphi_{n}\right)\right)
$$

The natural Hermitian inner product on $\mathbb{C}^{2 g-2}$ for which the basis $x_{i}, y_{i}$ is orthogonal induces an inner product $(\cdot, \cdot)$ on $V=\wedge^{g-1} \mathbb{C}^{2 g-2}$. We denote by $\|\cdot\|$ the corresponding norm, and we let $\|A\|$ be the operator norm of a matrix $A \in \mathrm{GL}(V)$ with respect to this norm.

We will use the following result by Benoist and Quint, which is a combination of Theorem 4.28(b) and Theorem 4.31 of [BQ16]. In its formulation, we use as before the special point $e=x_{1} \wedge \cdots \wedge x_{g-1} \in \wedge^{g-1} \mathbb{C}^{2 g-2}$.

Theorem 6.7. (The law of large numbers) For a fixed finitely supported probability measure $\mu$ on $\widehat{\mathcal{I}}_{g}^{(q)}$ and for fixed $q \geq 7$ or for $q=5$, there exists a number $\lambda=\lambda(\mu, q)>0$ such that for $\beta$-almost every $b \in B$, one has

$$
\frac{1}{n} \log \left\|\rho_{q}\left(b_{n} \cdots b_{1}\right) \cdot e\right\| \rightarrow \lambda
$$

Furthermore, this convergence also holds in $\mathrm{L}^{1}(B, \beta)$. That is, the functions $L_{n}: B \rightarrow \mathbb{R}$ defined by

$$
L_{n}(b)=\frac{1}{n} \log \left\|\rho_{q}\left(b_{n} \cdots b_{1}\right) \cdot e\right\|,
$$

converge in $\mathrm{L}^{1}(B, \beta)$ to the constant function $\lambda(\mu, q)$ as $n \rightarrow \infty$.

Proof. The results of Benoist and Quint are valid for any random walk on $\mathrm{GL}(V)$ whose support generates (as a semigroup) a subgroup $\Gamma$ of $\mathrm{GL}(V)$ so that the standard representation of $\Gamma$ on $V$ is strongly irreducible and proximal. That these properties hold true for the representations $\sigma_{q}$ was shown in Lemma 6.6.

It's important to stress here that $\lambda(\mu, q)$ depends only on $\mu$ and $q$, but not on the sample path $b \in B$.

The following result about random walks on projective spaces is also due to Benoist and Quint BQ14. In its formulation, convolution of measures in $\mathbb{P}\left(\mathbb{C}^{d}\right)$ is via the orbit map for the action of $\mathrm{GL}\left(\mathbb{C}^{d}\right)$. 
Proposition 6.8. BQ14, Theorem 1.1 (ii)] Let $\mu$ be a measure on $\mathrm{GL}\left(\mathbb{C}^{d}\right)$ such that the Zariski closure of the semi-group generated by the support of $\mu$ is semi-simple. Let $x \in \mathbb{P}\left(\mathbb{C}^{d}\right)$. Then the convolutions $\mu^{* n} \star \delta_{x}$ converge to a $\mu$-stationary measure $\nu$ on $\mathbb{P}\left(\mathbb{C}^{d}\right)$.

The following result is due to Gol'dsheld and Margulis GM89 (see also BQ16, Lemma 4.6]).

Proposition 6.9. Let $\mu$ be a probability measure on $\mathrm{GL}\left(\mathbb{C}^{d}\right)$. Let $\Gamma_{\mu}$ denote the smallest subsemigroup of $\mathrm{GL}\left(\mathbb{C}^{d}\right)$ such that $\mu\left(\Gamma_{\mu}\right)=1$. Let $\nu$ be a $\mu$ stationary probability measure on $\mathbb{P}\left(\mathbb{C}^{d}\right)$. If $\Gamma_{\mu}$ acts strongly irreducibly on $\mathbb{C}^{d}$ then $\nu(\mathbb{P}(W))=0$ for any proper linear subspace $W$ of $\mathbb{C}^{d}$.

6.5. Controlling Finite Covers. Recall that $\lambda(\mu, q)>0$ is the Lyapunov exponent associated to a finitely supported probability measure $\mu$ on $\widehat{\mathcal{I}}_{g}^{(q)}$ and a representation $\rho_{q}: \widehat{\mathcal{I}}_{g}^{(q)} \rightarrow \mathrm{U}(g-1, g-1$ ) (see Theorem 6.7). Furthermore, let $\nu=\lim _{n \rightarrow \infty} \mu^{\star n} \star \delta_{e}$ whose existence is guaranteed by Proposition 6.8,

Corollary 6.10. Let $q \geq 3$; then for every $\alpha \in(0, \lambda(\mu, q))$ we have:

$$
\mu^{* n}\left(\left\{\varphi \in \widehat{\mathcal{I}}_{g}^{(q)}||\left(\rho_{q}(\varphi) e, f\right) \mid<\exp (\alpha n)\right\}\right) \rightarrow 0
$$

as $n \rightarrow \infty$.

Proof. The claim will follow from two statements, namely:

I: For every $\varepsilon>0$ there exists a $\delta=\delta(\varepsilon)>0$ and an $n_{0}=n_{0}(\varepsilon) \in \mathbb{N}$ such that:

$$
\mu^{* n}\left(\left\{\varphi \in \widehat{\mathcal{I}}_{g}^{(q)} \mid \frac{\left|\left(\rho_{q}(\varphi) e, f\right)\right|}{\left\|\rho_{q}(\varphi) e\right\|}<\delta\right\}\right)<\varepsilon / 2,
$$

for all $n \geq n_{0}$.

II: For every $\varepsilon, \delta>0$ there exists an $n_{1}=n_{1}(\varepsilon, \delta) \in \mathbb{N}$ so that for all $n \geq n_{1}$ we have:

$$
\mu^{* n}\left(\left\{\varphi \in \widehat{\mathcal{I}}_{g}^{(q)} \mid\left\|\rho_{q}(\varphi) e\right\|<\exp (\alpha n) / \delta\right\}\right)<\varepsilon / 2,
$$

Assuming these for a moment, the claim is immediate: if $\left|\left(\rho_{q}(\varphi) e, f\right)\right|<$ $\exp (\alpha n)$ then

$$
\frac{\left|\left(\rho_{q}(\varphi) e, f\right)\right|}{\left\|\rho_{q}(\varphi) e\right\|}<\delta \text { or }\left\|\rho_{q}(\varphi) e\right\|<\exp (\alpha n) / \delta
$$

As such, combining I and II proves the corollary.

To prove $\mathbf{I}$, we note that the condition $(\cdot, f)=0$ defines a linear subspace of $\wedge^{g-1} \mathbb{C}^{2 g-2}$ of proper codimension. As such

$$
\nu\left(\left\{v \in \mathbb{P}\left(\wedge^{g-1} \mathbb{C}^{2 g-2}\right) \mid \frac{|(v, f)|}{\|v\|}=0\right\}\right)=0
$$


by Proposition 6.9, By regularity of $\nu$ and continuity of the function

$$
x \mapsto \frac{|(x, f)|}{\|x\|}
$$

on $\mathbb{P}\left(\wedge^{g-1} \mathbb{C}^{2 g-2}\right)$, there exists a number $\delta>0$ so that

$$
\nu\left(\left\{v \in \mathbb{P}\left(\wedge^{g-1} \mathbb{C}^{2 g-2}\right) \mid \frac{|(v, f)|}{\|v\|} \leq \delta\right\}\right)<\varepsilon / 4 .
$$

Therefore weak convergence of the measures $\mu^{* n} * \delta_{e}$ to $\nu$ implies

$$
\limsup _{n \rightarrow \infty} \mu^{* n} \star \delta_{e}\left(\left\{v \in \mathbb{P}\left(\wedge^{g-1} \mathbb{C}^{2 g-2}\right) \mid \frac{|(v, f)|}{\|v\|} \leq \delta\right\}\right)<\varepsilon / 4 .
$$

The inequality we are after for $n$ large enough now follows from the identity

$$
\begin{aligned}
& \mu^{* n} \star \delta_{e}\left(\left\{v \in \mathbb{P}\left(\wedge^{g-1} \mathbb{C}^{2 g-2}\right) \mid \frac{|(v, f)|}{\|v\|} \leq \delta\right\}\right) \\
&=\mu^{* n}\left(\left\{\varphi \in \widehat{\mathcal{I}}_{g}^{(q)} \mid \frac{\left|\left(\rho_{q}(\varphi) e, f\right)\right|}{\left\|\rho_{q}(\varphi) e\right\|} \leq \delta\right\}\right) .
\end{aligned}
$$

We will prove Claim II using the law of large numbers (Theorem 6.7). The $\mathrm{L}^{1}(B, \beta)$ convergence of the functions $L_{n}: B \rightarrow \mathbb{R}$ to the constant function $\lambda$ implies that as $n \rightarrow \infty$

$$
\int_{B}\left|L_{n}(b)-\lambda\right| d \beta(b) \rightarrow 0
$$

Let $\alpha^{\prime}>0$ so that $\alpha<\alpha^{\prime}<\lambda$. The convergence above tells us that for all $\varepsilon>0$ there exists an $n_{1} \in \mathbb{N}$ so that for all $n \geq n_{1}$ :

$$
\beta\left(\left\{b \in B \mid \frac{1}{n} \log \left\|\rho_{q}\left(b_{n} \cdots b_{1}\right) \cdot e\right\|<\alpha^{\prime}\right\}\right)<\varepsilon / 2 .
$$

Because the condition on the left is only a condition on the initial $n$ entries of the infinite path $b \in B$, using the relation between $\beta$ and $\mu$ described at the beginning of the section we have

$$
\beta\left(\left\{b \in B \mid L_{n}(b) \leq \alpha^{\prime} n\right\}\right)=\mu^{* n}\left(\left\{\varphi \in \widehat{\mathcal{I}}_{g}^{(q)} \mid \log \left\|\rho_{q}(\varphi) \cdot e\right\| \leq \alpha^{\prime} n\right\}\right) .
$$

By increasing $n_{1}$, we can make sure that $\exp \left(\alpha^{\prime} n\right)>\exp (\alpha n) / \delta$. This shows II.

6.6. The proof of the main theorems. Putting all the above together, we can now prove Theorem 6.1 .

The proof of Theorem 6.1. Let us denote by $\mathcal{B} \subset \mathcal{I}_{\mathrm{g}}$ the set of those elements of the Torelli group that do not have $E_{\sigma}$ (the condition we defined in the beginning of this section). For a number $\alpha>0$ (chosen below) let $K_{\alpha, \mu}$ be 
the finite set given by Proposition 6.3. The same proposition shows that for any $q \in \mathbb{N}$ we have

$$
\mathcal{B} \cap \operatorname{supp}\left(\mu^{* n}\right) \subset \mathcal{A}^{(n)} \cup \bigcup_{k \in K_{\alpha, \mu}} \mathcal{C}_{k}
$$

where

$$
\begin{gathered}
\mathcal{A}^{(n)}=\left\{\varphi \in \mathcal{I}_{\mathrm{g}}||\left(\rho_{q}(\hat{\varphi}) e, f\right) \mid<\exp (\alpha n) \text { for some lift } \hat{\varphi} \text { of } \varphi\right\}, \\
\left.\mathcal{C}_{k}=\left\{\varphi \in \mathcal{I}_{\mathrm{g}} \mid \operatorname{det}\left(B_{\infty}(\varphi)\right) \text { contains } \Phi_{k} \text { as a factor }\right)\right\}
\end{gathered}
$$

Set $q=3$ and assume that $\alpha<\lambda(\mu, 3)$ (in addition to further constraints below). Consider the projection $\pi: \widehat{\mathcal{I}}_{g}^{(3)} \rightarrow \mathcal{I}_{\mathrm{g}}$. By Lemma 6.4, we have

$$
\mu^{* n}\left(\mathcal{A}^{(n)}\right)=\zeta^{* n}\left(\pi^{-1} \mathcal{A}^{(n)}\right)
$$

where $\zeta$ is the lifted measure as in Section 6.2. Now,

$$
\zeta^{* n}\left(\pi^{-1} \mathcal{A}^{(n)}\right)=\zeta^{* n}\left(\left\{\hat{\varphi} \in \widehat{\mathcal{I}}_{g}^{(3)}||\left(\rho_{3}(\hat{\varphi}) e, f\right) \mid<\exp (\alpha n)\right\}\right)
$$

and hence as $\alpha<\lambda(\mu, 3)$, it follows from Corollary 6.10 that

$$
\mu^{* n}\left(\mathcal{A}^{(n)}\right)=\zeta^{* n}\left(\pi^{-1} \mathcal{A}^{(n)}\right) \rightarrow 0 \quad(n \rightarrow \infty) .
$$

As $K_{\alpha, \mu}$ is a finite set, for the proof of the theorem it now suffices to show that for each fixed $k \in K_{\alpha, \mu}$ we have $\mu^{* n}\left(\mathcal{C}_{k}\right) \rightarrow 0$ as $n \rightarrow \infty$.

Thus let $k \in K_{\alpha, \mu}$ and assume first that $k \geq 3$. If $\operatorname{det}\left(B_{\infty}(\varphi)\right)$ contains $\Phi_{k}$ as a factor, then Lemma 4.7 implies that $\left(\rho_{k}(\hat{\varphi}) e, f\right)=0$ for a lift $\hat{\varphi}$ of $\varphi$ to $S_{k}$. Now the equation $(\cdot, f)=0$ defines a proper linear subspace of $\wedge^{g-1} \mathbb{C}^{2 g-2}$, so arguing as in the proof of Corollary 6.10 we see that for a finitely supported probability measure $\zeta$ on $\widehat{\mathcal{I}}_{g}^{(k)}$ whose support generates $\widehat{\mathcal{I}}_{g}^{(k)}$ as a semigroup, we have that

$$
\zeta^{* n}\left(\left\{\tilde{\varphi} \in \widehat{\mathcal{I}}_{g}^{(k)} \mid\left(\rho_{k}(\hat{\varphi}) e, f\right)=0\right\}\right) \rightarrow 0
$$

Using again Lemma 6.4, this implies that

$$
\mu^{* n}\left(\mathcal{C}_{k}\right) \rightarrow 0
$$

for $k \geq 3$.

We are thus left with controlling the case where $\operatorname{det}\left(B_{\infty}(\varphi)\right)$ contains at least one of $\Phi_{1}, \Phi_{2}$ as a factor, but no other $\Phi_{k}$ with $k \in K_{\alpha, \mu}$. In other words, we need to show that

$$
\mu^{* n}\left(\left(\mathcal{C}_{1} \cup \mathcal{C}_{2}\right) \backslash \bigcup_{k \in K_{\alpha, \mu}, k \geq 3} \mathcal{C}_{k}\right) \rightarrow 0 .
$$

To this end, we will decompose the set in question into two sets $\mathcal{D}_{1}, \mathcal{D}_{2}$ and show that their measures converge to 0 . Namely, let $\mathcal{D}_{i} \subset \mathcal{I}_{\mathrm{g}}$ be subset of the Torelli group where

$$
\operatorname{det}\left(B_{\infty}(\varphi)\right)=\Phi_{1}^{m_{1}} \cdot \Phi_{2}^{m_{2}} \cdot Q
$$


for some polynomial $Q \in \mathbb{Z}[t]$ that does not contain $\Phi_{k}$ as a factor for any $k \in\{1,2\} \cup K_{\alpha, \mu}$ and $m_{1}, m_{2} \in \mathbb{N}$ are such that $m_{i} \geq m_{j} \forall j \neq i$ and $m_{i} \geq 1$. In other words, $\mathcal{D}_{i}$ is the set where of the two "problematic" polynomials $\Phi_{1}$ and $\Phi_{2}, \Phi_{i}$ appears with the largest exponent. Note that indeed

$$
\left(\mathcal{C}_{1} \cup \mathcal{C}_{2}\right) \backslash \bigcup_{k \in K_{\alpha, \mu}, k \geq 3} \mathcal{C}_{k}=\mathcal{D}_{1} \cup \mathcal{D}_{2}
$$

Note that there is a number $B>1$ so that

$$
\left|\Phi_{i}(\xi)\right| \leq B, \quad \forall i=1,2 \forall \xi \in \mathbb{C},|\xi|=1 .
$$

Since primitive roots of unity of prime order are dense in the unit circle, we can choose distinct primes $q_{i}>2$ and $q_{i}$-th roots of unity $\zeta_{q_{i}}$ so that

$$
\left|\Phi_{i}\left(\zeta_{q_{i}}\right)\right| \leq B^{-1} \quad \forall i=1,2
$$

and therefore

$$
\left|\Phi_{i}\left(\zeta_{q_{i}}\right)\right| \cdot \max \left\{1,\left|\Phi_{j}\left(\zeta_{q_{i}}\right)\right|\right\} \leq 1
$$

for $i \neq j \in\{1,2\}$. We will also assume from now on that the map $\iota$ : $\mathbb{Z}\left[\mathbb{Z} / q_{i} \mathbb{Z}\right] \rightarrow \mathbb{C}$ introduced in Section 4.2 maps the generator $1 \in \mathbb{Z} / q_{i} \mathbb{Z}$ to this chosen primitive $q_{i}$-th root of unity $\zeta_{q_{i}}$.

We further impose that $\alpha>0$ satisfies

$$
\alpha<\min \left\{\lambda\left(\mu, q_{1}\right), \lambda\left(\mu, q_{2}\right)\right\}
$$

Note that by our choices, using Proposition [5.4, for all $\varphi \in \mathcal{D}_{i}$ we have that for a lift $\hat{\varphi}$

$$
\left|\left(\rho_{q_{i}}(\hat{\varphi}) e, f\right)\right|=\left|\operatorname{det}\left(B_{\infty}(\varphi)\right)\left(\zeta_{q_{i}}\right)\right| \leq \exp (\alpha n)
$$

Namely,

$$
\begin{gathered}
\left|\operatorname{det}\left(B_{\infty}(\varphi)\right)\left(\zeta_{q_{i}}\right)\right|= \\
\left|\Phi_{1}^{m_{1}}\left(\zeta_{q_{i}}\right) \cdot \Phi_{2}^{m_{2}}\left(\zeta_{q_{i}}\right) \cdot Q\left(\zeta_{q_{i}}\right)\right| \leq\left|Q\left(\zeta_{q_{i}}\right)\right|
\end{gathered}
$$

and arguing as in the proof of Proposition 6.3 we have

$$
\left|Q\left(\zeta_{q_{i}}\right)\right| \leq \exp \left(\alpha^{\prime} \operatorname{deg}(Q)\right) \leq \exp \left(\alpha^{\prime} \operatorname{deg}\left(\operatorname{det}\left(B_{\infty}(\varphi)\right)\right)<\exp (\alpha n),\right.
$$

where $\alpha^{\prime}$ is the number computed from $\alpha$ in that proof.

Hence, since $q_{i}>2$, we can argue as in the case of the $\mathcal{A}^{(n)}$ to show that

$$
\mu^{* n}\left(\mathcal{D}_{i}\right) \rightarrow 0
$$

This finishes the proof of the theorem.

We conclude with the proof of the second main theorem. Namely,

Theorem 6.11. Let $\mu$ be any measure on $\mathcal{I}_{\mathrm{g}}$ (or a homology stabiliser) with finite support so that the support generates $\mathcal{I}_{\mathrm{g}}$ as a semigroup. Fix $d>0$. Then

as $n \rightarrow \infty$.

$$
\mu^{* n}\left(\left\{\begin{array}{l|l}
\varphi \in \mathcal{I}_{\mathrm{g}} \mid \begin{array}{c}
b_{1}\left(N_{\varphi}^{\prime}\right)>b_{1}\left(N_{\varphi}\right) \text { for some } \\
\text { Abelian cover } N_{\varphi}^{\prime} \text { of degree } \leq d
\end{array}
\end{array}\right\}\right) \rightarrow 0
$$


As a first reduction, note that it suffices to consider cyclic covers. This is due to the fact that every representation of an Abelian group factors through a cyclic group. See the discussion in [DT06, Section 9.3] for details on this reduction. As there are only finitely many such cyclic covers, it suffices to show the conclusion for one.

Fix a cyclic cover $S_{q} \rightarrow S$. Let $\widehat{N_{\varphi}}$ be the cover of $N_{\varphi}$ defined by $S_{q} \rightarrow S$.

Proposition 6.12. The cover $\widehat{N_{\varphi}}$ has strictly larger Betti number than $N_{\varphi}$ if and only if

$$
\iota\left(\operatorname{det} B_{q}(\varphi)\right)=0
$$

Proof. Recall that $E=\operatorname{ker}\left(H_{1}\left(S^{\prime} ; \mathbb{Z}\right) \rightarrow H_{1}(S ; \mathbb{Z})\right)$ and that $H_{1}\left(S^{\prime} ; \mathbb{Z}\right)=$ $\mathbb{Z}[G]^{2 g-2} \oplus \mathbb{Z}^{2}$. We define a map

$$
\mathcal{J}: H_{1}\left(S^{\prime} ; \mathbb{Z}\right) \rightarrow \mathbb{C}^{2 g-2}
$$

by setting it to be zero on the trivial summand $\mathbb{Z}^{2}$ and applying the map $\iota$ (from Section 4.2) coordinate-wise on $\mathbb{Z}[G]^{2 g-2}$. As the kernel of $\iota: \mathbb{Z}[G] \rightarrow$ $\mathbb{C}$ is exactly the trivial representation contained in $\mathbb{Z}[G]$, the homomorphism $\mathcal{J}$ induces an isomorphism of $E$ onto its image $\mathcal{J}(E)$. Consequently, the induced map $\mathcal{J}_{\mathbb{Q}}: H_{1}\left(S^{\prime} ; \mathbb{Q}\right) \rightarrow \mathbb{C}$ induces an isomorphism from $E \otimes \mathbb{Q}$ onto its image $\mathcal{J}_{\mathbb{Q}}(E \otimes \mathbb{Q})$

By Proposition 2.6, we have that $b_{1}\left(\widehat{N_{\varphi}}\right)>b_{1}\left(N_{\varphi}\right)$ if and only if

$$
\left(L_{E}^{\prime}+\widehat{\varphi}_{*} L_{E}^{\prime}\right) \otimes \mathbb{Q} \subsetneq E \otimes \mathbb{Q}
$$

is a proper subspace, or equivalently, if

$$
\iota\left(L_{E}^{\prime} \otimes \mathbb{Q}\right)+\iota\left(\widehat{\varphi}_{*} L_{E}^{\prime} \otimes \mathbb{Q}\right) \subset \iota(E \otimes \mathbb{Q})
$$

is a proper subspace. By definition of $B_{q}$, the latter is the case exactly if $\iota\left(\operatorname{det} B_{q}(\varphi)\right) \neq 0$. This shows the proposition.

Arguing as in the proof of Theorem 1, the asymptotic probability that the determinants det $B_{q}(\varphi)$ satisfy the (algebraic) condition from Proposition 6.12 will converge to 0 , proving Theorem 2 .

Remark 6.13. In order to extend [DT06, Theorem 9.1] to genus $g \geq 3$ using this method, one can argue as follows. As above, it suffices to consider cyclic covers. For a fixed cyclic cover, the subgroup $\Gamma$ of the mapping class group which does lift to the cover and so that lifts commute with the deck group action has finite index in the mapping class group. Standard equidistribution results for random walks on finite graphs can be used to show that the desired result is true for a random walk on the mapping class group if it is true for a random walk on $\Gamma$. On $\Gamma$ one can define the representation $\rho$ as before, and the argument given for Theorem 6.11 applies.

We end with a sketch of the following result, which addresses a question in [Ko08, p. 139]. 
Theorem 6.14. Let $\mu$ be a probability measure on $\operatorname{Mod}\left(S_{g}\right)$ whose finite support generates $\operatorname{Mod}\left(S_{g}\right)$ as a semigroup. Then there is an $\alpha>0$ so that

$$
\mu^{* n}\left(\left\{\varphi \in \operatorname{Mod}\left(S_{g}\right) \mid \#\left(H_{1}\left(N_{\varphi} ; \mathbb{Z}\right)_{\text {tors }}\right)<\exp (\alpha n)\right) \rightarrow 0\right.
$$

Sketch of proof. Consider the standard representation

$$
\rho: \operatorname{Mod}\left(S_{g}\right) \rightarrow \operatorname{Sp}(2 g, \mathrm{Z})
$$

on the homology of the surface. It is known that this is surjective. As in Section 3, one can define a "bottom-left block" $B(\varphi)$ of $\rho(\varphi)$ so that

$$
\#\left(H_{1}\left(N_{\varphi} ; \mathbb{Z}\right)_{\text {tors }}\right)=\operatorname{det} B(\varphi)
$$

supposing that the determinant $\operatorname{det} B(\varphi)$ is nonzero. Now, one can use the representation $\rho$ in place of the $\rho_{q}$ to let the mapping class group act on Lagrangian subspaces of $\mathbb{R}^{2 g}$.

Given a basis $v_{1}, \ldots, v_{g} \in \mathbb{R}^{2 g}$ of a Lagrangian subspace of $\mathbb{R}^{2 g}$, we obtain a vector $v_{1} \wedge \cdots \wedge v_{g} \in \wedge^{g} \mathbb{R}^{2 g}$. The action of $\operatorname{Sp}(2 g, \mathbb{Z})$ on the subspace $W \subset \wedge^{g} \mathbb{R}^{2 g}$ spanned by all vectors obtained from Lagrangian subspaces of $\mathbb{R}^{2 g}$ is known to be irreducible and proximal. As such the same results of Gol'dsherd and Margulis GM89] and Benoist and Quint BQ14, BQ16 that are used in Section 6.4 apply.

Thus one can show that, first, with probability converging to 1 , det $B(\varphi) \neq$ 0 (as the opposite is a proper subspace of $W$ ), and in fact it does grow exponentially fast with the length $n$ of the walk (by the law of large numbers).

\section{REFERENCES}

[BGS16] U. Bader, T. Gelander, R. Sauer, Homology and homotopy complexity in negative curvature, arXiv:1612.04871

[BV13] N. Bergeron, A. Venkatesh, The asymptotic growth of torsion homology for arithmetic groups. J. Inst. Math. Jussieu 12 (2013), no. 2, 391-447.

[BSV16] N. Bergeron, M. H. Sengun and A. Venkatesh, Torsion homology growth and cycle complexity of arithmetic manifolds, Duke Math. J. 165 (2016), 16291693.

[BQ14] Y. Benoist, J.-F. Quint, Random walks on projective spaces, Compos. Math. 150 (2014), no. 9, 1579-1606.

[BQ16] Y. Benoist, J.-F. Quint, Random walks on reductive groups, Springer Ergebnisse der Math. 62, Springer 2016.

[CF13] T. Church, B. Farb, Representation theory and homological stability, Advances in Mathematics 245 (2013), 250-314.

[CW34] C. Chevalley and A. Weil, Über das Verhalten der Integrale 1. Gattung bei Automorphismen des Funktionenkörpers. Abh. Math. Sem. Univ. Hamburg, 10:358361, 1934.

[DT06] N.M. Dunfield, W.P. Thurston, Finite covers of random 3-manifolds, Inventiones Mathematicae 166 (2006), pp. 457-521.

[EW] G. Everest, T. Ward, Heights of polynomials and entropy in algebraic dynamics, Universitext. Springer-Verlag London, Ltd., London, 1999.

[FM12] B. Farb, D. Margalit, A primer on mapping class groups, Princeton University Press, Princeton 2012.

[FH91] W. Fulton, J. Harris, Representation theory. A first course, Graduate Texts in Mathematics, 129. Springer Verlag, New York, 1991. 
[GM89] I. Ya. Gol'dshel̆d, G. A. Margulis, Lyapunov exponents of a product of random matrices, Uspekhi Mat. Nauk 44 (1989), 13-60.

[GS91] F. Gonzáles-Acuña, H. Short, Cyclic branched coverings of knots and homology spheres. Revista Math. 4 (1991) 97120.

[HO89] A.J. Hahn and O.T. O'Meara, The Classical Groups and K-Theory, Springer, Berlin and New York, 1989.

[HW08] G. H. Hardy, E. M. Wright, An introduction to the theory of numbers, Oxford University Press, Oxford, 2008.

[Hi06] S. Hirose, The action of the handlebody group on the first homology group of the surface. Kyungpook Math. J. 46 (2006), no. 3, 399-408.

[Kr57] L. Kronecker, Zwei Sätze über Gleichungen mit ganzzahligen Coefficienten. J. Reine Angew. Math. 53 (1857), 173-175.

[Ko08] E. Kowalski, The large sieve and its applications. Arithmetic geometry, random walks and discrete groups, Cambridge Tracts in Math. 175, Cambridge Univ. Press, Cambridge 2008.

[Le14] T. Le, Homology torsion growth and Mahler measure, Comm. Math. Helv. 89 (2014), 719-757.

[Li16] Y. Liu, Immersing Quasi-Fuchsian surfaces of odd Euler characteristic in closed hyperbolic 3-manifolds, arXiv:1603.07069

[LiS16] Y. Liu, H. Sun, Virtual 1-domination of 3-manifolds, arXiv:1610.03937

[Lo97] E. Looijenga, Prym Representations of Mapping Class Groups, Geometriae Dedicata 64 (1997), pp. 69-83.

[LMW14] A. Lubotzky, J. Maher, C. Wu, Random methods in 3-manifold theory, arXiv:1405.6410

[Ma10] J. Maher, Random Heegaard splittings, J. Topol. 3 (2010), 997-1025.

[Ma96] H. Maier, The size of the coefficients of cyclotomic polynomials. Analytic number theory, Vol. 2 (Allerton Park, IL, 1995), Progr. Math. 139, Birkhuser Boston, Boston, MA, (1996), pp. 633-639

[Ra12] J. Raimbault, Exponential growth of torsion in Abelian coverings, Algebraic and Geometric Topology 12, no. 3 (2012), pp. 1331-1372.

[Ri90] R. Riley, Growth of order of homology of cyclic branched covers of knots, Bull. London Math. Soc. 22 (1990) 287297.

[Ri14] I. Rivin, Statistics of 3-manifolds occasionally fibering over the circle, arXiv: $1401.5736 \mathrm{v} 4$.

[SW02a] D. S. Silver, S. G. Williams. Torsion numbers of augmented groups with applications to knots and links. Ens. Math. 48 (2002).

[SW02b] D. S. Silver, S. G. Williams. Mahler measure, links and homology growth. Topology, 41(5):979-991, 2002.

[S15a] H. Sun, Virtual homological torsion of closed hyperbolic 3-manifolds, J. Diff. Geom. 100 (2015), 547-583.

[S15b] H. Sun, Virtual domination of 3-manifolds, Geom. Top. 19 (2015), 2277-2328.

\section{Current affiliations of the authors:}

Hyungryul Baik (hrbaik@kaist.ac.kr)

DEPARTMENT OF MATHEMATICAL SCIENCES, KAIST

291 DAEHAK-RO, YUSEONG-GU, DAEJEON, 34141, REPUBLIC OF KOREA 
David Bauer (david-bauer@uni-bonn.de)

Ursula Hamenstädt (ursula@math.uni-bonn.de)

Sebastian Hensel (hensel@math.uni-bonn.de)

Thorben Kastenholz (tkastenholz@gmx.de)

Bram Petri (bpetri@math.uni-bonn.de)

Daniel Valenzuela (daniel@valenzuela.de)

MATHEMATISCHES INSITUT DER UNIVERSITÄT BONN

ENDENICHER ALLEE 60, 53115 BONN

GERMANY

Ilya Gekhtman (ilya.gekhtman@yale.edu)

DEPARTMENT OF MATHEMATICS, YALE UNIVERSITY

10 HILLHOUSE AV, NEW HAVEN, CONNECTICUT 06520

USA 